\title{
To gauge or not to gauge?
}

\author{
Juan Maldacena ${ }^{a}$ and Alexey Milekhin ${ }^{b}$ \\ ${ }^{a}$ School of Natural Sciences, Institute for Advanced Study, \\ 1 Einstein Drive, Princeton, NJ, U.S.A. \\ ${ }^{b}$ Physics Department, Princeton University, \\ Jadwin Hall, Washington Road, Princeton, NJ, U.S.A. \\ E-mail: malda@ias.edu, milekhin@princeton.edu
}

ABSTRACT: The D0 brane, or BFSS, matrix model is a quantum mechanical theory with an interesting gravity dual. We consider a variant of this model where we treat the $\mathrm{SU}(N)$ symmetry as a global symmetry, rather than as a gauge symmetry. This variant contains new non-singlet states. We consider the impact of these new states on its gravity dual. We argue that the gravity dual is essentially the same as the one for the original matrix model. The non-singlet states have higher energy at strong coupling and are therefore dynamically suppressed.

Keywords: AdS-CFT Correspondence, D-branes, Gauge Symmetry, M(atrix) Theories

ARXIV EPRINT: 1802.00428 


\section{Contents}

$\begin{array}{llr}1 & \text { Introduction } & 2\end{array}$

2 The Do brane matrix model 3

2.1 The matrix model 3

2.2 The gravity dual 5

2.3 The size of the matrix versus the size of the Einstein gravity region 6

3 The ungauged model $\quad 6$

$\begin{array}{ll}3.1 \text { Lack of supersymmetry } & 7\end{array}$

3.2 Supersymmetric version of the ungauged model 8

3.3 Relation to Wilson loop insertions 8

4 Gravity duals of non-singlets $\quad 9$

$\begin{array}{ll}4.1 & \text { Exploring the large } X \text { region }\end{array}$

4.2 Adjoint energies at weak coupling in the BMN matrix model 11

$\begin{array}{lll}4.3 & \text { Spectrum above the minimum } & 13\end{array}$

$\begin{array}{ll}4.4 \text { The free energy } & 15\end{array}$

5 Deconfinement and the eigenvalues Polyakov loop holonomy 16

6 Further comments $\quad 19$

6.1 Is there a bulk $\mathrm{SU}(N)$ gauge field associated to the $\mathrm{SU}(N)$ global symmetry $\begin{array}{ll}\text { of the ungauged model? } & 19\end{array}$

$\begin{array}{ll}\text { 6.2 } & \text { Are there gauge fields on brane probes? } \\ \end{array}$

$\begin{array}{ll}6.3 & \text { The ungauged model and M-theory } 20\end{array}$

$\begin{array}{lll}6.4 & \text { Physical realizations } & 20\end{array}$

$\begin{array}{lll}7 & \text { Conclusions } & 21\end{array}$

A Details of the perturbative computations $\quad 22$

A.1 Non singlets in the BMN matrix model 22

A.2 BFSS model 24

$\begin{array}{lll}\text { A.2.1 Solving the potential for a uniform distribution } & 27\end{array}$

A.3 Goldstone modes and $\mathrm{SU}(N)$ rotators for the BMN model vacua 28

B Analyzing the motion of a folded string $\quad 30$

C Scaling properties of the solution and the action 32 


\section{Introduction}

Many examples of the holographic correspondence involve very strongly coupled large $N$ gauge theories which are dual to a bulk Einstein gravity theory [1-3]. In such theories, the gauge symmetry leads to a reduction in the naive number of low dimension operators from $N^{2}$ to an order one number. The D0 brane matrix model [4], also known as BFSS model [5], is an example of such gauge/gravity duality [6]. In a $0+1$ dimensional theory, the only role of the gauge symmetry is to impose an $\mathrm{SU}(N)$ singlet constraint. Therefore, we can consider an alternative model where we set $A_{t}=0$. The theory now has a global $\mathrm{SU}(N)$ symmetry. If we impose a "Gauss Law" constraint restricting to $\mathrm{SU}(N)$ singlets, then we recover the gauged model. In this paper we study the properties of the model where we do not impose this singlet constraint.

At first sight, one might think that not imposing this constraint leads to many more states, of order $N^{2}$ of them. The presence of these new states could modify the properties of the system substantially. This is indeed correct in the weakly coupled regime. However, we will argue that in the strongly coupled regime we have essentially the same gravity dual description as for the gauged model.

In this matrix model, the coupling constant, $g^{2}$, has dimensions of (mass) ${ }^{3}$. Therefore it is weakly coupled at high energies and strongly coupled at low energies. Correspondingly, the gravity dual has a curvature that depends on the radial position. Near the boundary it is highly curved, but away from the boundary we have a low curvature region where we can trust Einstein gravity. See figure 1. This low curvature region corresponds to the energy scales where the matrix model is strongly coupled.

We will argue/conjecture that the gravity picture of the non-singlet states is the following. The non-singlet states have an energy of order the order $\lambda^{1 / 3}=\left(g^{2} N\right)^{1 / 3}$ and are located in the high curvature region, away from the region that is described by Einstein gravity, see figure 1. In the planar approximation, we also have states corresponding to additional excitations of these non-singlet states which can be represented as folded strings with their ends stuck to the highly curved region near the boundary. At finite temperature we can further have non-singlet states that correspond to black holes with strings that come in from the boundary and end on the black hole, see figure 2(b), 2(c).

We have not derived this picture rigorously, we will simply present some plausibility arguments and consistency checks.

In [7], Berkowitz, Hanada, Rinaldi and Vranas present more evidence supporting this picture by performing a direct numerical simulation of the ungauged model. ${ }^{1}$

In [8], a similar conclusion was reached for the "double scaling" limit of a single matrix quantum mechanics for low enough temperatures See also [9-13] for further discussion of non-singlets in that model.

We were motivated to ask the question in the title by the Gurau-Witten tensor models $[14,15]$. There one has the choice of either imposing or not imposing a singlet constraint. It is sometimes thought that the models with a singlet constraint would be more likely to have a gravity or string dual. Our main message is that the existence of a local gravity dual

\footnotetext{
${ }^{1}$ We thank the authors of [7] for detailed explanations on their computations and for ongoing discussions.
} 
is independent of whether we do or do not impose this constraint. Einstein-like gravity is associated to very strong interactions but not to the presence or absence of the boundary theory gauge symmetry (or gauge redundancies).

When we consider the ungauged model we break supersymmetry, since in the original model the algebra only closes up to gauge transformations. Nevertheless the modified algebra can be used to argue that the energy is positive, even for non-singlet states. Of course, singlet states are the same as those of the gauged model. For non-singlet states, the lowest energy state appears to be when all branes are separated by a large amount (namely, the matrices get large diagonal expectation values). In this regime, the non-zero $\operatorname{SU}(N)$ charges lead to a kind of angular potential going like $1 / X^{2}$ for diagonal matrices of typical magnitude $X$. This potential leads to even larger expectation values for the matrices. Nevertheless, for finite temperatures, we expect to have a metastable state where the expectation values of the matrices are relatively small (or the branes are together), since this state has more entropy. This state can be viewed as a black hole. Our previous remarks on the equality of the gravity configurations applied for these metastable black hole configurations.

We are arguing that non-singlets are energetically disfavored at low energies. This seems to contradict the picture proposed in $[16,17]$ for the deconfinement/black hole transition that is based on the idea that the Polyakov loop gets an expectation value. If the only states contributing were singlets we would get no potential for the eigenvalues of the Polyakov loop. We will discuss how the two pictures are consistent. We are lead to a picture where the values of the holonomy indeed break the center symmetry but only by a very small amplitude "wave" in the eigenvalue distribution.

This paper is organized as follows. In section 2 we review the BFSS matrix model and its holograhic dual. Also we describe its massive deformation, the BMN or plane wave model. In section 3 we describe how one can remove the singlet constaint and obtain the deformation of the supersymmetry algebra. We also relate it to the insertion of Wilson loops in the gauged model. Section 4 is devoted to non-singlets. We first look at the lowest energy excitations of the thermal blackground. We then consider the region where all branes are far away. We also use perturbation theory to find the shifts to the spectrum in the weakly coupled region. We also discuss the thermodynamic properties of the ungauged model. We discuss further aspects of the Polyakov loop and thermal phase transitions in section 5. After making some further comments we present some conclusions.

\section{The Do brane matrix model}

In this section we review the D0 brane matrix model and its gravity dual. Readers familiar with this material can jump directly the next section.

\subsection{The matrix model}

The D0 matrix model [4], or BFSS matrix model [5], has the action

$$
S=\frac{1}{g^{2}} \int d t \operatorname{Tr}\left(\frac{1}{2}\left(D_{t} X^{I}\right)^{2}+\frac{1}{2} \psi_{\alpha} D_{t} \psi_{\alpha}+\frac{1}{4}\left[X^{I}, X^{J}\right]^{2}+\frac{1}{2} i \psi_{\alpha} \gamma_{\alpha \beta}^{I}\left[\psi_{\beta}, X^{I}\right]\right)
$$


where all indices are summed over. $I, J=1, \cdots, 9, \alpha, \beta=1, \cdots 16$. where $\gamma^{I}$ are nine dimensional gamma matrices which are real, symmetric and traceless. ${ }^{2} \psi_{\alpha}$ are hermitian $N \times N$ matrices, which can be expanded as $\psi_{\alpha}=\psi_{\alpha}^{r} T^{r}$ where the $T^{r}$ are a complete set of hermitian $N \times N$ matrices, and we can think of the $r$ index as a real index of the adjoint representation of $\mathrm{U}(N)$. Then $\psi_{\alpha}^{r}$ are Majorarana fermions. We have $16 \times N^{2}$ Majorana fermions. This model is invariant under 16 supersymmetries and also under an $\mathrm{SO}(9)$ R-symmetry.

The model has a $\mathrm{U}(N)$ gauge symmety and the derivative is defined as $D_{t} B=\partial_{t} B+$ $i\left[A_{t}, B\right]$ where $A_{t}$ is the gauge field. We could choose the gauge where $A_{t}=0$ and then we have to impose Gauss's law:

$$
G=\frac{i}{2 g^{2}}\left(2\left[D_{t} X^{I}, X^{I}\right]+\left[\psi_{\alpha}, \psi_{\alpha}\right]\right)=0 .
$$

It restricts all states to be singlets under the $\mathrm{U}(N)$ symmetry.

Classical zero energy configurations correspond to simultaneously diagonal matrices $X^{I}$. Quantum mechanically, the model has a zero energy bound state. At finite temperature it is expected (from the gravity picture) to have a metastable bound state.

It is interesting to ask what the typical size of the matrices $X^{I}$ is in the ground state or in a thermal state. This was estimated [18] by setting a lower bound for $\frac{1}{N} \operatorname{Tr}\left[X^{2}\right]$, using virial theorem ideas. That lead to

$$
\sqrt{\left\langle\frac{1}{N} \operatorname{Tr}\left[X^{2}\right]\right\rangle} \sim \lambda^{1 / 3}, \quad \lambda \equiv g^{2} N
$$

In a heuristic way, this can also be obtained by dimensional analysis and large $N$ counting if one assumes that $\lambda$ is the only relevant scale (and not the temperature). This result will be particularly useful when we analyze the gravity solution.

There is a variant of this model where we add mass terms that break $\mathrm{SO}(9) \rightarrow \mathrm{SO}(3) \times$ $\mathrm{SO}(6)$ [19]. The additional terms in the action are

$$
\begin{aligned}
S_{\mathrm{BMN}}=S_{[\text {from }(2.1)]} & +S_{\mu}, \\
S_{\mu}=-\frac{1}{g^{2}} \int d t & \operatorname{Tr}\left(\frac{1}{2}\left(\frac{\mu}{3}\right)^{2} \sum_{a=1}^{3}\left(X^{a}\right)^{2}+\frac{1}{2}\left(\frac{\mu}{6}\right)^{2} \sum_{i=4}^{9}\left(X^{i}\right)^{2}+\frac{\mu}{8} \psi \gamma_{123} \psi\right. \\
& \left.+i \frac{\mu}{3} \sum_{a, b, c=1}^{3} X^{a} X^{b} X^{c} \epsilon_{a b c}\right) .
\end{aligned}
$$

It also preserves 16 supercharges but with a different supersymmetry algebra, SU(2|4). We can view (2.4) as a collection of harmonic oscillators and Majorana fermions with some particular interactions.

The mass terms remove the flat directions in the potential. Apart from the simplest vacuum with $X^{I}=0$, the BMN model also has additional vacua [20-22], characterized by non-zero $X^{a}, a=1,2,3$ such that:

$$
i \epsilon_{a b c} X^{b} X^{c}=\frac{\mu}{3} X^{a} .
$$

\footnotetext{
${ }^{2}$ We can view them as coming from the ten dimensional Majorana Weyl representation $\gamma^{I}=\Gamma^{0} \Gamma^{I}$.
} 
This equation is solved by $X^{a}=\frac{\mu}{3} J^{a}$, where $J^{a}$ are $\mathrm{SU}(2)$ algebra generators in an $N$ dimensional representation, not neccessarily irreducible. Such solutions represent a collection of fuzzy spheres. Although this vaccum breaks $\mathrm{SU}(N)$ symmetry, there are no physical Goldstone bosons because of the gauge symmetry. We will return to $\mathrm{SU}(N)$ Goldstone bosons later in section 3 when we discuss the ungauged model.

\subsection{The gravity dual}

We will be mostly discussing the gravity dual at finite temperature. The geometry is a solution of ten dimensional type IIA supergavity closely related to the near horizon geometry of a charged black hole in ten dimensions [23]. It is given by [6]

$$
\begin{aligned}
\frac{d s^{2}}{\alpha^{\prime}} & =-\frac{f_{0}(r) r^{7 / 2}}{\sqrt{\lambda d_{0}}} d t^{2}+\sqrt{\frac{\lambda d_{0}}{r^{3}}}\left(\frac{1}{f_{0}(r) r^{2}} d r^{2}+d \Omega_{8}^{2}\right) \\
e^{\phi} & =\frac{(2 \pi)^{2}}{d_{0}} \frac{1}{N}\left(\frac{\lambda d_{0}}{r^{3}}\right)^{7 / 4} \\
\tilde{A}_{t} & =\frac{N}{(2 \pi)^{2}} \frac{r^{7}}{\lambda^{2} d_{0}} \\
f_{0}(r) & =1-\frac{r_{0}^{7}}{r^{7}}, \quad d_{0} \equiv 240 \pi^{5}, \quad \lambda \equiv g^{2} N,
\end{aligned}
$$

where $r_{0}$ and the inverse temperature $\beta=1 / T$ are related by ${ }^{3}$

$$
\frac{1}{T}=\beta=\frac{4}{7} \pi \sqrt{\lambda d_{0}} r_{0}^{-5 / 2} .
$$

This geometry has an effective radius of curvature given by the radius of $S^{8}$

$$
\frac{R_{\text {eff }}^{2}}{\alpha^{\prime}}=\sqrt{\frac{\lambda d_{0}}{r^{3}}}
$$

which is a function of the radial direction. For this reason we can trust (2.7) only in some region of the geometry, namely $r \lesssim \lambda^{1 / 3}$. Note that $r$ has units of energy. At larger values of $r$, when $\lambda^{1 / 3} \lesssim r$, the curvature is high and we cannot trust the gravity solution. The large $r$ region is where the boundary is and it corresponds to the UV of the boundary theory. In this region the matrix model is weakly coupled and we can trust perturbation theory.

The geometry at the horizon of the black hole will be weakly curved as long as

$$
1 \ll \lambda \beta^{3} .
$$

There is an additional $N$ dependent constraint $\lambda \beta^{3} \ll N^{10 / 7}$ on the validity of this IIA supergravity solution that arises when we also demand that the dilaton is not too large at the horizon. In this paper, we will imagine that we are in the 't Hooft limit where $N$ is

\footnotetext{
${ }^{3}$ We can think of the relation between $\beta$ and $r_{0}$ as a way to translate between time scales in the matrix model $(\beta)$ and radial position in the bulk $\left(r_{0}\right)[24]$.
} 
taken to be very large compared to other quantities, such as $\lambda$ or $\beta$, or more precisely $\lambda \beta^{3}$. So we do not have to worry about this second constraint.

Using the Bekenstein-Hawking formula one can easily find the entropy and free energy ${ }^{4}$

$$
\begin{aligned}
& S=N^{2} 4^{13 / 5} 15^{2 / 5}(\pi / 7)^{14 / 5}\left(\frac{T}{\lambda^{1 / 3}}\right)^{9 / 5} \approx 11.5 N^{2}\left(\frac{T}{\lambda^{1 / 3}}\right)^{9 / 5} \\
& F=N^{2} \lambda^{1 / 3} \frac{5}{14} 4^{13 / 5} 15^{2 / 5}(\pi / 7)^{14 / 5}\left(\frac{T}{\lambda^{1 / 3}}\right)^{14 / 5} \approx 7.4 N^{2} \lambda^{1 / 3}\left(\frac{T}{\lambda^{1 / 3}}\right)^{14 / 5} .
\end{aligned}
$$

These predictions were checked in an increasingly sophisticated set of numerical computations [25-30] culminating in [31], where also the leading $\alpha^{\prime}$ corrections were computed. ${ }^{5}$

The gravity dual for the BMN case is a bit more complicated, it has some gapped states described in [32] and a black hole thermal state which looks like a deformation of (2.7) [33]. The magnitude of the deformation involves $\mu / T$ and it is very small if $\mu / T$ is small.

\subsection{The size of the matrix versus the size of the Einstein gravity region}

It is interesting to translate (2.3) to the gravity side. On the gravity side we can consider D0 brane probes that sit at particular values of $r$. A string stretching from this brane probe to $r=0$, or the horizon, has an energy of the order of $r$. Now, if we consider the mass of an off-diagonal mode of a matrix in the diagonal background $m \sim X_{\text {diag }}$ we expect to get the same energy. It means that the radial direction is related to matrix elements as $r \sim X$. Using this procedure to translate between radial positions and matrix eigenvalues, we now ask: what value of $r$ would the scale (2.3) correspond to? Interestingly, it corresponds to a scale $r \sim \lambda^{1 / 3}$, which is the scale at which the supergravity solution breaks down! This important point was emphasized in [18], and we are repeating it because we think it is not widely appreciated. In fact, some papers in the literature seem to suggest that the typical size of the matrices in the thermal state would be $X \sim r_{0}$. Note that $r_{0} \ll \lambda^{1 / 3}$ in the region where we can trust gravity.

This means that the whole Einstein gravity region of figure 1 corresponds to a highly quantum region of the wavefunction for the matrix model. The matrices have large fluctuations. However, these fluctuations are highly correlated. Indeed, via supersymmetric localization, [34, 35] computed $\frac{1}{N}\left\langle\operatorname{Tr}\left[\left(X^{1}+i X^{9}\right)^{2 k}\right]\right\rangle$. They found a much smaller answer agreeing with naive bulk expectations. Due to the $i$, in this expectation values there are interesting cancellations.

\section{$3 \quad$ The ungauged model}

In this paper we will consider the situation where we set $A_{t}=0$ and we do not impose the $\mathrm{SU}(N)$-singlet constraint. ${ }^{6}$ This amounts to treating the $\mathrm{SU}(N)$ symmetry as a global

\footnotetext{
${ }^{4}$ The temperature dependence can be recovered from the properties of (2.7) under rescalings. Namely sending $t \rightarrow \eta t$ and $r \rightarrow \eta^{-2 / 5} r$ the metric gets rescaled by an overall factor and the action by $S \rightarrow \eta^{-9 / 5} S$, which is also the scaling of the entropy. See appendix C.

${ }^{5}$ It is an interesting challenge to match the first correction by computing the full tree level $\alpha^{\prime 3}$ corrections to the tree level IIA supergravity in the effective action.

${ }^{6}$ We could also say that we have a $\mathrm{U}(N)$ gauge symmetry. However, since there are no fields charged under the overall $\mathrm{U}(1)$, it does not matter whether we gauge or do not gauge the overall $\mathrm{U}(1)$.
} 


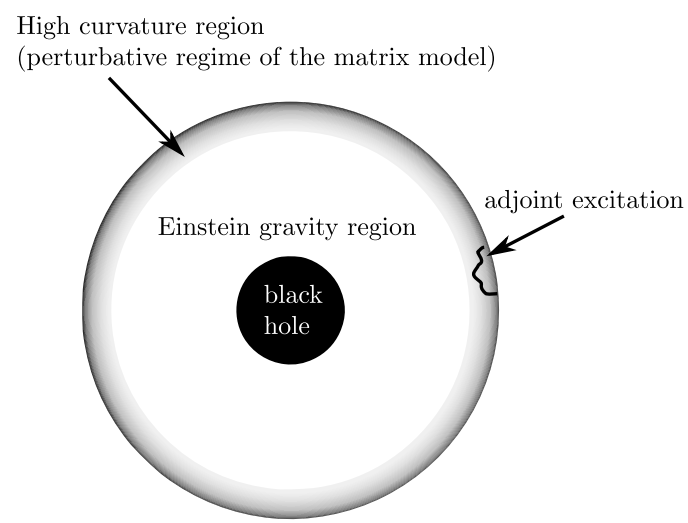

Figure 1. Sketch of the gravity solution at finite temperature. The shaded region near the boundary is highly curved. Moving further inwards we find a region of lower curvature that can be described by Einstein gravity. We will argue that the lowerst energy non-singlet excitations live purely in the shaded region and have an energy that roughly corresponds to that of a massive string state located in the interface between the two.

symmetry rather than as a gauge symmetry. In higher dimensions, gauging a symmetry introduces extra degrees of freedom. In quantum mechanics it does not. The theory with $A_{t}=0$ is a perfectly well defined theory, with global $\mathrm{SU}(N)$ symmetry, and we can consider it in its own right. This theory has a singlet subsector where it identical to the usual one in section 2, but it also has non-singlet states whose interpretation in the gravity dual we want to ellucidate.

It is sometimes said that gauging the $\mathrm{SU}(N)$ symmetry reduces the number of operators drastically and that this is important for the gravity solution to work. We will see that the gravity solution can be valid whether we gauge the $\mathrm{SU}(N)$ symmetry or not.

\subsection{Lack of supersymmetry}

Let us define the hamiltonian of the ungauged model to be simply the one obtained from eq. (2.1) by setting $A_{t}=0$. We can then wonder whether the resulting theory is supersymmetric. We certainly continue to have the operators $Q_{a}$ that were generating the SUSY transformations before:

$$
Q \epsilon=-\frac{1}{g^{2}} \operatorname{Tr}\left(\dot{X}^{I} \psi \gamma^{I} \epsilon+i \frac{1}{2}\left[X^{K}, X^{L}\right] \psi \gamma^{K L} \epsilon\right)
$$

where $\gamma^{K L}=\frac{1}{2}\left(\gamma^{K} \gamma^{L}-\gamma^{L} \gamma^{K}\right)$. We can now ask whether these operators commute with the Hamiltonian. We find

$$
\left[Q_{\alpha}, H\right]=-\operatorname{Tr}\left(\psi_{\alpha} G\right) .
$$

We see that the right hand side can be written in terms of the $\mathrm{SU}(N)$ symmetry generators, $G$ in (2.2). This means that, while (3.2) vanishes when it acts on singlet states, it will be non-vanishing acting on non-singlet states. Therefore we expect that non-singlets will not come in supersymmetry multiplets. We can also compute the anticommutators

$$
\left\{Q_{\alpha}, Q_{\beta}\right\}=2 H \delta_{\alpha \beta}+2 \operatorname{Tr}\left(G X^{I}\right) \gamma_{\alpha \beta}^{I} .
$$


We see that we get non-zero answers in the right hand side because the supersymmetry transformations only close up to $\mathrm{SU}(N)$ transformations. In the gauged model these are gauge transformations. But in the ungauged model we get a non-zero right hand side. Nonetheless, we can still infer some information from this algebra.

Let us note first, that even for non-siglet states the energy is non-negative. Indeed, since the supercharges are self-adjoint $Q_{\alpha}^{\dagger}=Q_{\alpha}$ and gamma matrices are traceless, summing over the spinorial indices leads to

$$
32 H=\sum_{\alpha=1}^{16}\left\{Q_{\alpha}, Q_{\alpha}\right\}=\sum_{\alpha=1}^{16}\left\{Q_{\alpha}^{\dagger}, Q_{\alpha}\right\} \geq 0 .
$$

\subsection{Supersymmetric version of the ungauged model}

In principle, we could modify the definition of the supercharges so as to have a supersymmetric theory. We do not think that is possible. Nevertheless, if we are willing to also redefine the Hamiltonian, then it is possible to preserve some of the supersymmetry. This can be achieved by adding a new term to the Hamiltonian:

$$
H_{\text {susy }}=H-\operatorname{Tr}\left(X^{1} G\right) \text {. }
$$

This breaks the $\mathrm{SO}(9)$ symmetry to $\mathrm{SO}(8)$, and it preserves half of the supersymmetry, those whose spinorial parameter obeys

$$
\left(\gamma^{1}+\mathbf{1}\right) \epsilon=0 .
$$

Moreover, now we have the standard supersymmetry algebra:

$$
\left\{Q \cdot \epsilon, Q \cdot \epsilon^{\prime}\right\}=2 H_{\text {susy }} \epsilon \cdot \epsilon^{\prime} .
$$

This might seem surprising at first sight, but there is a simple explanation for the existence of this Hamiltonian. In this paper we will concentrate on the model with the original Hamiltonian.

\subsection{Relation to Wilson loop insertions}

There is a physical situation that arises in the gauged model which is very closely connected to the ungauged model. We can have the original gauged theory and add an external quark in some representation $\bar{R}$, by coupling it through a Wilson line operator in representation $\bar{R}$. This is very closely related to the ungauged theory restricted to the representation $R .^{7}$ The only difference is that in the thermal partition function, restricted to representation $R$, we would include a factor of the dimension of the representation in the ungauged case but not in the gauged case with a Wilson loop.

The simplest Wilson loop operators we can consider are $\operatorname{Tr}_{\overline{\mathrm{R}}} P e^{i \int A_{t} d t}$. These break supersymmetry. Another commonly considered operator preserves half of the supersymmetries and has the form $\operatorname{Tr}_{\overline{\mathrm{R}}} P e^{i \int d t\left(A_{t}+X^{1}\right)}$, where we have picked one of the scalar

\footnotetext{
${ }^{7}$ We can only consider representations transform trivially under the $Z_{N}$ center of $\mathrm{SU}(N)$, which are the ones we can get from products of adjoints.
} 


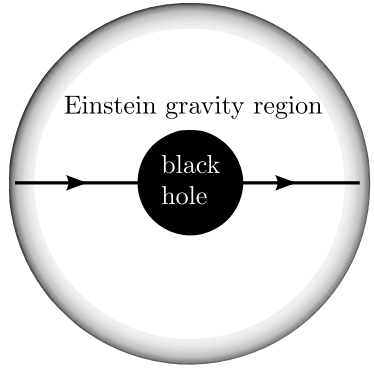

(a)

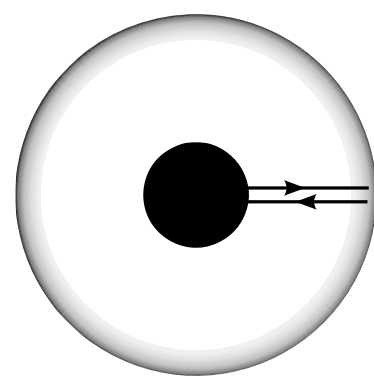

(b)

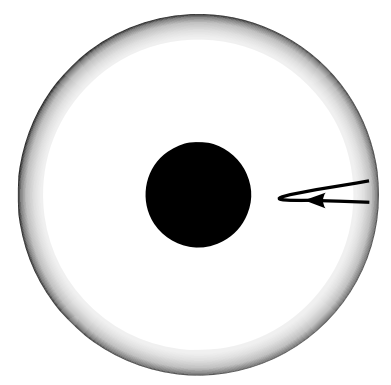

(c)

Figure 2. (a): the string configuration corresponding to a supersymmetric Wilson line in the adjoint representation. (b): the string and anti-string configuration representing a nonsupersymmetric adjoint. We get it from (a) by moving the left string segment to the right side of the figure. (c): we further lower the energy configuration of (b) by moving the tip away from the horizon. The idea is that the end point of this motion is a configuration as in figure 1.

fields $[36,37]$. The extra term corresponds to the extra term in the Hamiltonian (3.5). When we add the supersymmetric Wilson loop in the adjoint representation, in the gravity dual we get a string coming in from the boundary at $X^{1}=\infty$ and a string going to $X^{1}=-\infty$. Equivalently, we can say we have a string anti-string pair with the string pinned on the north pole of the $S^{8}$ and the anti-string on the south pole of $S^{8}$. See figure 2(a).

In conclusion, we can translate many of the statements in this paper to statements about insertions of Wilson lines for the original, gauged, model.

\section{Gravity duals of non-singlets}

Let us consider first the gravity dual of the adjoint states, states in the adjoint representation of $\mathrm{SU}(N)$. They are described by the gravity dual of the non-supersymmetric Wilson loop $e^{i \int A_{t} d t}$. As pointed out in [38] (see also [39]), the gravity dual of these Wilson loops differs from the supersymmetric Wilson loops only through the fact that the strings are not pinned at a particular point on the sphere, but they can move to any point on the sphere. See figure 2(b), 2(c). In other words, on the boundary of the bulk they obey Neumann, rather than Dirichlet, boundary conditions in the sphere directions. If we have an adjoint, this means that the string and the anti-string could lower their energy by coming closer together on the sphere. If they coincide on the sphere, then we have a folded string whose energy can be lowered by moving the tip further and further to the boundary, see figure 2(c).

According to the gravity solution, the energy of folded streteched string (at $\beta=\infty$ ), with its tip momentarily at rest, is given by

$$
E=\frac{2}{2 \pi} \int_{r_{\text {tip }}}^{r_{\infty}} d r=\frac{1}{\pi}\left(r_{\infty}-r_{\text {tip }}\right)
$$

This computation, valid in the gravity regime, would suggest that we can lower the energy to zero by moving $r_{\text {tip }} \rightarrow r_{\infty}$, where $r_{\infty}$ is some large $r$ cutoff. However, at very large 
$r$ we cannot trust the gravity computation. In other words, the fact that as $r_{\text {tip }} \rightarrow r_{\infty}$ the energy goes to zero cannot be trusted when both of these quantities are in the highly curved region. Therefore, it could be that even the lowest energy configuration has a nonzero energy. What would be a natural value for this energy? One natural possibility would be to think that $r_{\infty} \propto \lambda^{1 / 3}$ which is the value of $r$ where the curvature becomes of the order of the string scale. Furthermore, we can also assume that naive cancellation between $r_{\text {tip }}$ and $r_{\infty}$ does not happen and that we get an energy that is the typical energy of a massive string state at the location given by $r \sim \lambda^{1 / 3}$. From (2.7) we find that this is an energy of the order of $\lambda^{1 / 3}$. We get the same answer if we use dimensional analysis and assume that it will be of the order of the 't Hooft coupling. In both cases we get

$$
E_{\min }=C \lambda^{1 / 3}
$$

where $C$ is an unknown numerical constant. In the next subsection we will present an argument saying that $C>0$. Note that $C$ cannot be negative because we have argued near (3.4) that the energy should be positive. The fact that $C$ is positive is also suggested by the numerical computation in [7].

We can speculate about the temperature corrections to the estimate (4.2). We expect these to come from the fact that at finite temperature the metric at the transition region, at $r \sim \lambda^{1 / 3}$, will be changed due to the $r_{0}$ dependent terms in (2.7). We expect this to produce an extra factor of $\left(1+a_{1} \frac{r_{0}^{7}}{r^{7}}\right)$, where $r \sim \lambda^{1 / 3}$. Using (2.8) we find then that

$$
E=C \lambda^{1 / 3}\left(1+\tilde{a}_{1}\left(\frac{T^{3}}{\lambda}\right)^{\frac{14}{15}}+\cdots\right)
$$

where $\tilde{a}_{1}$ is an unknown numerical constant. The main point is that it is small for $T \ll \lambda^{1 / 3}$.

\subsection{Exploring the large $X$ region}

In the above discussion we have assumed that the model starts in a state with $X \sim 0$ and then we add the adjoint as a perturbation. This is particularly reasonable if the branes are trapped near the origin by thermal effects.

On the other hand, we can set the temperature to zero and consider a situation where all branes are separated from each other. In this case, we can ask about the energy of the adjoint state. First we should note that if we do not gauge the symmetry, then we have a manifold of Goldstone modes coming from applying the $\mathrm{SU}(N)$ transformations to the original configurations. This manifold has an $\mathrm{SU}(N)$ symmetry and we can consider a wavefunction which is in the adjoint representation under this global $\mathrm{SU}(N)$ symmetry. We can think of this as a configuration which has an $\mathrm{SU}(N)$ "angular momentum" along this manifold. The typical radius of this manifold is given by the distance between the branes, call it $X$. Then we get an energy which goes like

$$
V \sim+\frac{\lambda}{X^{2}}
$$

We discuss and derive this in more detail in appendix A.2. One can view this final formula as analogous to the angular momentum potentials we get when a particle moves in three 
dimensions in a spherically symmetric potential and with some angular momentum. It makes sense to first freeze $X$ and then calculate the potential (4.4) for the following reason. The effective mass of the $X$ variables is of order $1 / g^{2} \sim \frac{N}{\lambda}$, which is large in the 't Hooft limit. Therefore, the motion in the $X$ directions produced by (4.4) will be relatively slow. This is like the Born-Oppenheimer approximation. We can trust (4.4) when $|X|$ is large enough that we can use perturbation theory in the matrix model. This means that $\lambda / X^{3} \ll 1$. If we extend this to the boundary of its regime of validity, namely to $X^{3} \sim \lambda$, then we find that the energy becomes $\sim \lambda^{1 / 3}$, in agreement with the estimate (4.2). Figure 3 shows the form of the potential when we separate the branes and we are in an adjoint state. The reason we get a constant when $|X| \lesssim \lambda^{1 / 3}$ is the picture we suggested in figure 1 where the adjoint is localized in the transition region. When the branes are located within the Einstein gravity region they have shed their adjoint charge, leaving it as a string with endpoints in the high curvature region.

Note that this transition happens at a value of $r$ that coincides with the size of the ground state wave function (2.3). This also suggests that when the $X$ have an expectation value of this size there will be other degrees of freedom that can carry the adjoint quantum numbers.

Note that the presence of the potential in figure 3 suggests that the adjoint state with $X \sim 0$ is unstable and the system is driven to $X \sim \infty$. We think that this is the ultimate fate of adjoint states. On the other hand, at finite temperature the gravity solution shows that thermal effects will trap the branes at $X \sim 0$, leading to a metastable minimum. As we will recall near (4.16), this metastable state is very long lived in the 't Hooft limit, so that we only need to worry about this decay mode at very low temperatures.

\subsection{Adjoint energies at weak coupling in the BMN matrix model}

The BFSS matrix model is always strongly coupled at low energies. On the other hand, the BMN matrix model has another scale, given by the mass $\mu$. If we take $\lambda \ll \mu^{3}$, we can trust perturbative computations even around the simplest, $X=0$, vacuum. The expansion parameter is $\lambda / \mu^{3}$. In the simplest vacuum we have a collection of bosonic and fermionic harmonic oscillators. The lightest sector with matrix creation-annihilation operators $a_{i}^{\dagger}, a_{i}, i=1, \ldots, 6$ corresponds to $\mathrm{SO}(6)$ operators $X^{i}=i, i=1, \ldots, 6$, see appendix A.1 for details. Each oscillator $a_{i}^{\dagger}$ has energy $\mu / 6$. In the gauged $\mathrm{SU}(N)$ model we cannot act with a single creation operator because it would be in the adjoint of $\operatorname{SU}(N)$. The first singlet appears for a pair of operators $\operatorname{Tr}\left[a_{i}^{\dagger} a_{j}^{\dagger}\right]|0\rangle$, where the trace is over the $\mathrm{SU}(N)$ indices $r, s: \operatorname{Tr}\left[a_{i}^{\dagger} a_{j}^{\dagger}\right]=\left(a_{i}^{\dagger}\right)_{r}{ }^{s}\left(a_{j}^{\dagger}\right)_{s}{ }^{r}$. On the other hand, in the ungauged model we can have a state of the form $a_{i}^{\dagger}|0\rangle$. This state has energy $\mu / 6$ at zero coupling. One can compute the first perturbative correction and we find that it is given by (see appendix A.1)

$$
E_{\text {adjoint }}=\frac{\mu}{6}+\frac{9}{2} \frac{\lambda}{\mu^{2}}+\cdots
$$

where the dots are higher order terms in the $\lambda / \mu^{3}$ expansion. The idea is that if we were to sum all the corrections and continue the answer to strong coupling we would get that $E_{\text {adj }} \sim C \lambda^{1 / 3}$ for $\lambda / \mu^{3} \gg 1$. 


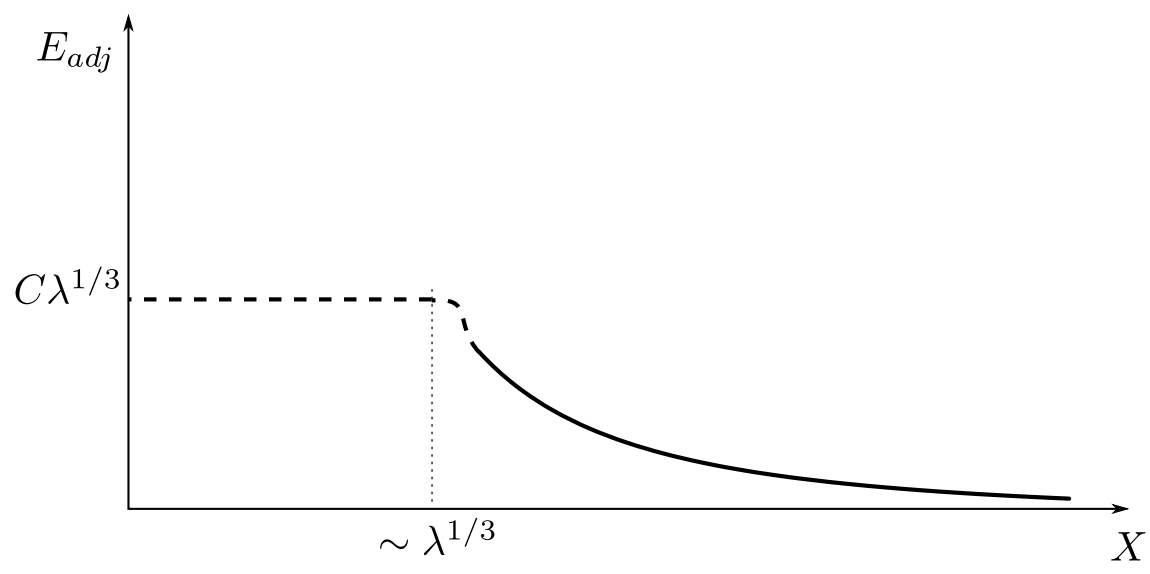

Figure 3. Energy of an adjoint state where we explore the flat directions of the potential in (2.1), which correspond to mutually diagonal matrices $X^{I}$. The solid line denotes the potential computed in the region where we can trust perturbation theory in the matrix model side, which is $\lambda^{1 / 3} \lesssim|X|$. The details depend on the particular form of the diagonal matrices $X$. In this region, the energy comes from the angular momentum along the $\mathrm{SU}(N)$ directions in the moduli space of vacua of the ungauged model. The horizontal doted line corresponds to the energy of a massive string state in the transition region, as in figure 1 . We expect a smooth transition region in between. We have not included here the effects of the thermal potential which produces a large dip near $X \sim 0$, or $r \sim r_{0}$, (for $T \ll \lambda^{1 / 3}$ ) because it is common to the gauged and ungauged models.

It is interesting that one can obtain a relatively simple answer for this one loop shift for the energy of more general adjoint states. This can be done using the general expression for the one loop Hamiltonian in [22] and observing that it contains an additional piece for non-singlets in representation $R$

$$
\Delta \hat{H}_{1-\text { loop }}=\Delta \hat{H}_{\text {gauged, 1-loop }}+\frac{9 g^{2}}{2 \mu^{2}} C_{2}(R) .
$$

The explicit expressions for $\Delta \hat{H}_{1 \text {-loop }}$ and $\Delta \hat{H}_{\text {gauged, 1-loop }}$ are given in eq. (A.14).

In general one should be careful with translating (4.6) to the energy shifts, since

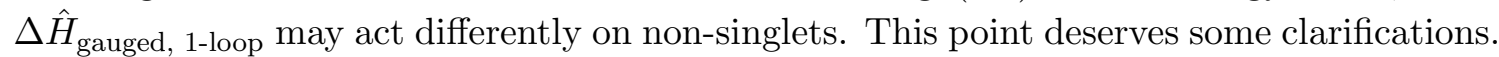
Since the trace is cyclic, operators $a_{i}^{\dagger}$, forming a single-trace singlet operator $\operatorname{Tr}\left[a_{i}^{\dagger} a_{j}^{\dagger} \ldots\right]$ are placed on "a circle". From this point of view, non-singlets have "boundaries". So, generically, singlets and non-singlets have quite different Hilbert spaces and $\Delta \hat{H}_{\text {gauged, 1-loop }}$ may have completely different eigenvalues.

For example, in the gauged model on level two we have BPS states $\operatorname{Tr}\left[a_{i}^{\dagger} a_{j}^{\dagger}\right], i \neq j$. However, in the adjoint sector of the ungauged model operators $a_{i}^{\dagger} a_{j}^{\dagger}$ and $a_{j}^{\dagger} a_{i}^{\dagger}$ are different if $i \neq j$. One can check explictly using eq. (A.14) that the state corresponding $a_{i}^{\dagger} a_{j}^{\dagger}+a_{j}^{\dagger} a_{i}^{\dagger}$

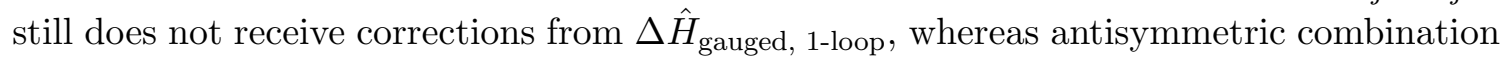
$a_{i}^{\dagger} a_{j}^{\dagger}-a_{j}^{\dagger} a_{i}^{\dagger}$ receives an additional shift of $4 g^{2}\left(\frac{3}{\mu}\right)^{2}$. Nonetheless, both symmetric and anti-symmetric combinations get contribution from $\frac{9 g^{2}}{2 \mu^{2}} C_{2}(\operatorname{adj})=\frac{9 g^{2} N}{2 \mu^{2}}$. The reason the symmetric combination is still protected against $\Delta \hat{H}_{\text {gauged, 1-loop }}$ is that symmetrization 
restores the cyclic symmetry. It is natural to conjecture that cyclic-symmetric non-singlets receive the same contribution from $\Delta \hat{H}_{\text {gauged, 1-loop }}$ as singlets.

So far we have discused the vacuum with $X=0$. One can also consider a fuzzy sphere vacuum (2.6) with $X^{a}=\frac{\mu}{3} J^{a}, a=1,2,3$. In this case, one also expects $\mathrm{SU}(N)$ Goldstone bosons. As in the discussion in section 4.1, we could calculate the energy of states with non-trivial $\mathrm{SU}(N)$ quantum numbers by considering states with $\mathrm{SU}(N)$ angular momentum along the manifold spanned by the Goldstone bosons. We discuss this in more detail in appendix A.3 where we found a simple lower bound on the energy of the adjoint of the form

$$
\frac{g^{2} N}{R^{2}}=\frac{\lambda}{R^{2}} \lesssim E_{\text {adjoint }}, \quad \text { for } \quad \mu^{3} \gg \lambda
$$

where $R^{2}=\frac{1}{3 N} \frac{\mu^{2}}{9} \operatorname{Tr}\left(J_{1}^{2}+J_{2}^{2}+J_{3}^{2}\right)$ is the average radius of the fuzzy spheres. This is consisent with the expectations based on (4.4).

\subsection{Spectrum above the minimum}

We now return to strong coupling. Around the thermal background we have argued that the minimum energy for the adjoint state is given in (4.2). We would now like to discuss excitations above these states. In the planar limit these excitations will be single strings of operators with a fundamental index at one end and an antifundamental at the other end, combined so that we an adjoint index in total. We expect that these states would be strings whose ends are located in the high curvature region.

Of course, when we quantize the string we expect a large number of modes. So we expect a number of energy eigenstates above the minimum given by (4.2). The first few are expected to be separated from the minimum by gaps which are of the same order of magnitude as the lowest energy itself (4.2). In general, it seems complicated to determine this spectrum because it depends both on what is happening in the high curvature region as well as in the low curvature region. As the string gets more excited it can dip further into the region described by Einstein gravity. An example of an excitation would be a stretched folded string that goes from the high curvature region to some radial position $r_{\min }$. If $r_{\min }$ is within the weakly coupled region, then its motion could be as indicated in figure 5, namely the tip of the string goes into the weakly coupled region, it is slowed down by the string that pulls it from the boundary and it bounces back to the high curvature region. ${ }^{8}$ After it goes back into the high curvature region it could come back out with other worldsheet excitations, depending on the physics in the high curvature region. The full spectrum cannot be obtained unless we can solve both parts of the motion, namely the one in the low curvature region as well as the one in the high curvature region. In appendix B we discuss a toy problem where we assume that the string tip is reflected from the high curvature region without any further excitation, thought this is probably not what happens in reality.

When the excitation energy is large enough that the string can reach all the way to the horizon, something new happens. The string falls into the horizon and we end up with

\footnotetext{
${ }^{8}$ We compute the phase shift for this motion in appendix B. A similar computation for the single matrix model in the double scaling limit was done in $[12,13]$ and matched to the matrix model.
} 
a string and an anti-string pair, each ending on the horizon. The minimum energy when this happens is given by the energy of a folded string that stretches all the way from the high curvature region to the horizon,

$$
E_{\mathrm{dec}}=\frac{1}{\pi} \int_{r_{0}}^{r_{\mathrm{high} \sim \lambda^{1 / 3}}} d r=\tilde{C} \lambda^{1 / 3}-\frac{r_{0}}{\pi}=\lambda^{1 / 3}\left[\tilde{C}-\frac{1}{\pi}\left(\frac{T 4 \pi \sqrt{d_{0}}}{7 \lambda^{1 / 3}}\right)^{\frac{2}{5}}\right]
$$

where we expect that $\tilde{C}$ is an order one quantity bigger than $C$ in (4.2).

We will call this the "deconfinement" energy, because above this energy the adjoint is effectively behaving as two independent excitations, a quark and an anti-quark, corresponding to the string and antistring segments ending on the horizon. Furthermore, when a string ends on the horizon, there is an additional factor $N$ in the effective number of states. This arises as follows. When a string wraps the Euclidean black hole it has a disk topology, which produces and additional factor of $1 / g_{s} \propto N$. This is in addition to the factor of $N$ that we get from all the possible values of the fundamental index at the boundary. This new factor is present for both the gauged and ungauged models and it is related to the physics at the horizon.

When the string is not reaching the black hole horizon we can effectively think of the large $N$ Hilbert space as factorizing into the black hole part which lives in the singlet Hilbert space and non-singlet excitations that live close to the boundary.

$$
\mathcal{H}_{\text {non-singlets }} \sim \mathcal{H}_{\text {singlets }} \otimes \mathcal{H}_{\text {boundary string Fock space }} \text {. }
$$

Furthermore there is a Fock space of boundary excitations, generated by the adjoint excitations which appear as strings with ends in the large curvature region, as in figure 1. Each generator has the degeneracy of an adjoint, or a factor of $N^{2} .9$

The strings that end on the horizon can be qualitatively viewed as extra tensor factors, one for the quarks and one for the anti-quarks (or strings or anti-strings), see figure 2(b). Each of these generates a Fock space. The string ending on the horizon is expected to have minimum energy $\tilde{C} \lambda^{1 / 3} / 2$. The same is true for the anti-string. This is because the folded string whose tip is at the horizon has energy $\tilde{C} \lambda^{1 / 3}$ by definition. And this is becoming the string/anti-string pair. The degeneracy of each generator also is proportional to $N^{2}$ but with a temperature dependent factor that can be computed by considering a string wrapping the black hole, which has an extra free energy given by (4.8) plus a logarithm of $N$, related to the factor of $1 / g_{s}$ in the partition function mentioned above. This extra degeneracy is not exact, it simply reflects an increase in the enropy of the combined black hole and string system, but we do not expect to be able to separate it cleanly into a black hole part and a string part. We get the following schematic decomposition of the Hilbert space

$$
\mathcal{H}_{\text {non-singlets }} \sim \mathcal{H}_{\text {singlets }} \otimes \mathcal{H}_{\text {boundary string Fock }} \otimes \mathcal{H}_{\text {horizon string Fock }} \otimes \mathcal{H}_{\text {horizon antistring Fock }}
$$

\footnotetext{
${ }^{9}$ We are idenfiying $N^{2}-1 \sim N^{2}$ since we are only discussing the leading $N$ effects.
} 


\subsection{The free energy}

In this subsection we consider the free energy of the ungauged theory. Because gauging is removing of order $N^{2}$ degrees of freedom, and given that the free energy is of order $N^{2}$, one might worry that the free energy of the gauged model would be very different than that of the ungauged one.

In fact, large $N$ counting tells us that

$$
-\beta F_{\text {ungauged }}-\beta F_{\text {gauged }}=N^{2} f\left(\lambda^{1 / 3} \beta\right) .
$$

For simplicity we could start considering the BMN model at weak coupling. In this case, in the ungauged theory we basically have $9 N^{2}$ bosonic harmonic oscillators, while in the gauged theory we have $8 N^{2}$ bosonic oscillators since the gauge constraint is essentially removing one matrix (the one we can diagonalize). The fermions give a subleading contribution in this high temperature limit. Therefore, in this case we get

$$
f \sim-\log (\mu \beta), \quad \lambda \beta^{3} \ll 1, \quad \frac{\lambda}{\mu^{3}} \ll 1 .
$$

On the other hand, at strong coupling, $\lambda \beta^{3} \gg 1 \gg \beta \mu$, where we can trust the black hole solution, we have a different picture. The idea is that non-singlets are extra adjoint particles living near the boundary of the gravity solution. Because of the factorization (4.10) they contribute with extra factors of the form

$$
\left(1+N^{2} d_{\text {Adj }} e^{-\beta \lambda^{1 / 3} C}\right)
$$

in the partition function. This is the contribution of just the lowest energy adjoint state and $d_{\text {Adj }}$ is its degeneracy. We expect it to be of order one. The factor of $N^{2}$ comes from the dimension of the adjoint representation. Therefore we expect that the leading energy difference is

$$
\beta F_{\text {gauged }}-\beta F_{\text {ungauged }}=N^{2} d_{\text {Adj }} e^{-\beta \lambda^{1 / 3} C}, \quad \lambda \beta^{3} \gg 1 .
$$

This shows that $F_{\text {gauged }}$ and $F_{\text {ungauged }}$ are exponentially close in the strongly coupled limit, while they are different at weak coupling (4.12).

Let us emphasize that at strong coupling we have a reduction in the naively expected number of states in both theories. For that reason one might have thought that the gauging or not gauging would have a large impact. However, we see that this is not what is happening, both theories have a common low energy description.

Using the factorized from of the Hilbert space, we can write a more precise form for the free energy difference

$$
f \sim \sum_{n}-(-1)^{F} \log \left[1-(-1)^{F} e^{-\beta E_{n}}\right]
$$

where $n$ runs over all the adjoint states which can be bosons or fermions. This follows from standard large $N$ counting.

We can further improve the discussion by including strings ending at the horizon. These contributions are most clear in Euclidean space. They still give contributions to $f$ that are exponentially suppressed $\propto e^{-\beta \lambda^{1 / 3} \tilde{C}}$. These are smaller than (4.14) because $C<\tilde{C}$. 
We have mentioned in the introduction that both ungauged and gauged models are unstable at very low temperatures. Here we will review more precise estimates for the decay rates (see eg. [40]). Let us start from the gauged model. Emitting a single D0 brane to infinity will lower the Bekenstein-Hawking entropy (2.11). Therefore such process is suppresed by:

$$
P \sim \exp \left(-\frac{\partial S}{\partial N}\right)=\exp \left(-2 \frac{S}{N}\right)
$$

where $S$ is given in (2.11). The instability is unsuppressed when

$$
T_{c} \sim \frac{\lambda^{1 / 3}}{N^{5 / 9}}
$$

Formally, at this temperature the dilaton becomes large at the horizon and one has to lift the gravity solution (2.7) to 11d M-theory black string [6]. Generically, black strings suffer from the Gregory-Laflamme instability [41], which, in this case, also occurs at the temperature (4.17).

The contribution from the lowest adjoint (4.14) will enchance (4.16) by $\sim e^{-\beta \lambda^{1 / 3} C}$. However at $T_{c}$ this factor is extremely small $e^{-N^{5 / 9}}$. Therefore the instability in the ungauged model occurs at the same temperature. Indeed, as we have mentioned before, this instability is the instability of the black hole itself, so excitations near the boundary should not affect it.

\section{Deconfinement and the eigenvalues Polyakov loop holonomy}

The main point of this paper is that in theories with gravity duals all non-singlets have high energies and are not dynamically important at low energies. On the other hand, the arguments in $[16,17]$ seem to suggest that non-singlets are important for modifying the eigenvalue distribution of the Polyakov loop. Furthermore, the fact that this distribution is not uniform is viewed as a signal of a black hole formation in the bulk.

This seems to be in contradiction with what we are saying, since we are emphasizing that the non-singlets are dynamically unimportant at low energies and strong coupling. We will here show why there is no contradiction.

To start, let us suppose that we are studying the gauged model. Then the partition function includes the integral over the gauge field holonomy, which we can take to diagonal $U=\operatorname{diag}\left(e^{i \theta_{1}}, \ldots, e^{i \theta_{N}}\right)$. In the large $N$ limit it is convenient to introduce the normalized density function $\rho(\theta)$

$$
\int_{-\pi}^{\pi} d \theta \rho(\theta)=1
$$

and the corresponding moments $\rho_{n}=\int_{-\pi}^{\pi} d \theta e^{i n \theta} \rho(\theta)$. The moments $\rho_{n}$ measure the nonhomogenity of the density function.

Since we only have adjoint fields in the matrix model, the energy can depend only on the relative distance between the eigenvalues $\theta_{i}-\theta_{j}$. There is a constant repulsion of order one among eigenvalues $\theta_{i}$ due to the group measure. Integrating out the matter fields leads to an attraction of eigenvalues. At very low temperatures the repulsions dominates and 
the density function is uniform (in the BMN model). As the temperature increases, the attraction becomes stronger and stronger until the density function jumps to a non-uniform distribution. In other words, eigenvalues from a cluster [17]. However, since the energy depends on the relative distance only, the absolute position of the cluster is not fixed, and one has to integrate over this zero mode. This is the reason why the Polyakov loop in the fundamental representation is still zero after the transition.

This resembles the gravity computation of the Polyakov loop in the fundamental [16]: one can have a single string stretched between the horizon and infinity. Such a string has a finite action and one could expect that the Polyakov loop will not be zero. However, in the black hole background one has a normalizable mode of the 2 -form $B_{\mu \nu}$, which couples to the string as

$$
\exp (i b), \quad b \equiv \int B
$$

And after the integration over $b$ one gets zero.

If we have just a single adjoint particle of mass $C \lambda^{1 / 3}$ and degeneracy $d_{\text {Adj }}$, then the partition function reads as (see [17] for the derivation):

$$
Z=\int d \rho_{1} \exp \left(-N^{2}\left|\rho_{1}\right|^{2}\left[1-d_{\mathrm{Adj}} e^{-\beta C \lambda^{1 / 3}}\right]\right)
$$

where in the exponent we have ignored the small terms proportional to $e^{-2 \beta C \lambda^{1 / 3}}, e^{-3 \beta C \lambda^{1 / 3}}$ and so on. The first term in brackets, the one, comes from the $\mathrm{SU}(N)$ measure, whereas the second term comes from the matter contribution, where $\left|\rho_{1}\right|^{2}$ is the contribution of the trace of the holonomy in the adjoint representation.

Assuming that $d_{\mathrm{Adj}}$ and $C$ are of order 1 and $\beta \lambda^{1 / 3} \gg 1$, the integral is dominated by $\rho_{1}=0$. Then, the density is uniform and we expect no black hole! This would be the right conclusion if the only states we had were the ones corresponding to strings with both ends at the high curvature region. For example, these are the only non-singlets around the gapped vacua of the BMN model in the strong coupling region.

However, apart from those strings, we can also have strings ending on the black hole. These strings effectively behave as quarks and antiquarks, with an overall constraint that there is an equal number of quarks and anti-quarks. We can view the integral over $b$ in (5.2) as enforcing this constraint. Therefore we can now write a partition function of the form

$$
Z=\int d^{2} \rho_{1} d b \exp \left(-N^{2}\left[\left|\rho_{1}\right|^{2}-d^{\prime} e^{-\beta \lambda^{1 / 3} \tilde{C} / 2}\left(e^{i b} \rho_{1}+e^{-i b} \bar{\rho}_{1}\right)\right]\right)
$$

where $d^{\prime}$ is a temperature dependent quantity that is less important than the exponential factor we are explicitly writing. We will discuss the origin of $d^{\prime}$ below. We now see that, before integrating over $b$, the integral does have a non-trivial saddle point for $\rho_{1}$

$$
\rho_{1}^{s}=e^{-i b} d^{\prime} e^{-\beta \lambda^{1 / 3} \tilde{C} / 2} .
$$

Higher $\rho_{n}, n \geq 2$ are suppressed by factors of $\left(e^{-\beta \lambda^{1 / 3} \tilde{C} / 2}\right)^{n}$. It means that the density $\rho(\theta)$ has a bump determined by the cosine function, see figure 4 . Of course, in this discussion we used the gravity solution to say what answer to expect on the matrix model side. We 


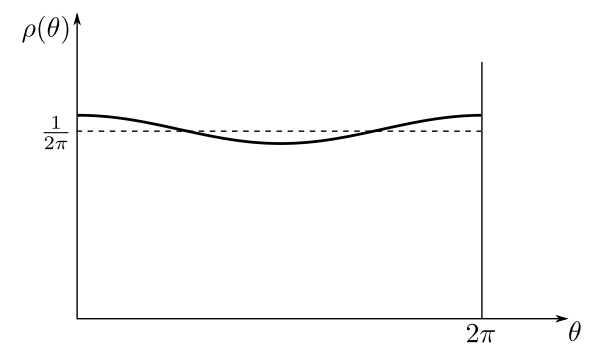

Figure 4. The eigenvalue density of the Polyakov loop at strong coupling. It is only slightly non-uniform.

have not derived this directly from the matrix model side! We are simply spelling out what answer we expect.

We have a non-zero VEV of the Polyakov loop in the adjoint representation:

$$
\left\langle W_{\text {adj }}\right\rangle=\left\langle\operatorname{Tr}_{\text {adj }} P e^{i \int A}\right\rangle=N^{2}\left|\rho_{1}^{s}\right|^{2} \propto N^{2} e^{-\beta \lambda^{1 / 3} \tilde{C}} .
$$

In other words, the large energy required to stretch a string from the boundary to the horizon implies that the expectation value of this Wilson loop is very small. Of course, the Polyakov loop in the fundamental is still zero since we have an integral over $b$.

Let us say a few words about the pre-exponent factor $d^{\prime}$. The combination $d^{\prime} e^{-\beta \lambda^{1 / 3} \tilde{C} / 2}$ in eq. (5.4) comes from a single string streched between the boundary at $r=\tilde{r}_{\infty}$ and the horizon at $r=r_{0}$. Therefore,

$$
d^{\prime} \exp \left(-\beta \lambda^{1 / 3} \tilde{C} / 2\right)=\mathcal{D} \exp \left(-\beta \frac{\tilde{r}_{\infty}-r_{0}}{2 \pi}\right) .
$$

The prefactor $\mathcal{D}$ arises from the one loop integral around the classical string configuration, which is a string that wraps the radial and Euclidean time directions. Due to the scaling properties of the solution (2.7), it can only give a power law term in the temperature. The exponential term $\beta \frac{\tilde{r}_{\infty}-r_{0}}{2 \pi}$ arises from the classical string action. As before, we expect that $\tilde{r}_{\infty}$ is a cutoff dependent quantity that is independent of the temperature, which we write as $\tilde{r}_{\infty} / \pi=\tilde{C} \lambda^{1 / 3}$. This constant is expected to be larger that $C$ in (4.2) since a string/anti-string pair ending on the horizon can decay into the massive string modes that live at the high curvature regions, which have the energy (4.2). On the other hand the $r_{0}$ term gives a temperature dependent term in the exponent

$$
\frac{\beta r_{0}}{2 \pi}=\frac{\beta^{3 / 5}}{2 \pi}\left(\frac{4 \pi \sqrt{\lambda d_{0}}}{7}\right)^{2 / 5}
$$

where we have used eq. (2.8) to find $r_{0}$ in terms of $\beta$.

The adjoint particles that live near the high curvature region (see figure 1) contribute as $e^{-\beta \lambda^{1 / 3} C}$ to the expectation value in (5.6), which is suppressed by $1 / N^{2}$ compared to (5.6), but the exponential suppression is smaller, since $C<\tilde{C}$. So the full expectation value in the adjoint is

$$
\left\langle W_{\text {adj }}\right\rangle \propto N^{2} e^{-\beta \lambda^{1 / 3} \tilde{C}}+e^{-\beta \lambda^{1 / 3} C}+\cdots
$$




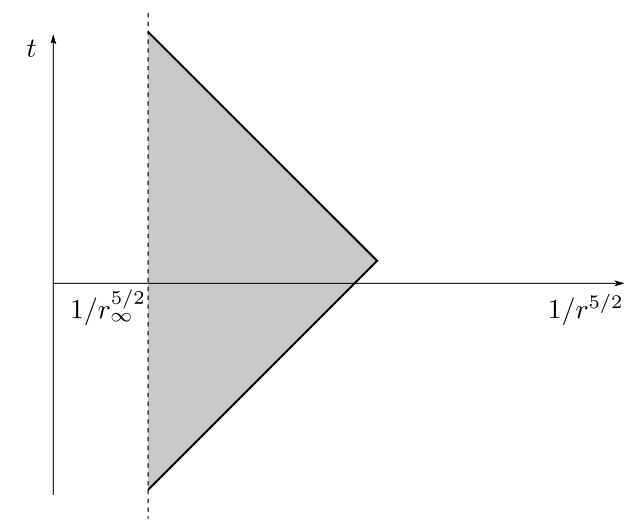

Figure 5. Radial excitations of the adjoint string. The string tip moves close to a null-geodesic (bold line). $r_{\infty} \sim \lambda^{1 / 3}$ is the region where the high curvature region starts. We imagine that when the string reaches that point it bounces back with some reflection factor.

where the dots refer to other contributions described by strings that are not ending at the horizon. The order $N^{0}$ contribution can be viewed as arising from doing the Gaussian integral in (5.3). In matrix model vacua with no black holes (such as the $X=0$ vacuum of the BMN matrix model) we only get the second term in (5.9).

\section{$6 \quad$ Further comments}

\subsection{Is there a bulk $\mathrm{SU}(N)$ gauge field associated to the $\mathrm{SU}(N)$ global sym- metry of the ungauged model?}

The ungauged model has a global $\mathrm{SU}(N)$ symmetry. According to the usual expectations, this should correspond to an $\mathrm{SU}(N)$ gauge symmetry in the bulk. On the other hand, we have argued that the bulk theory, at least in the Einstein gravity region, is essentially the same as that of the gauged model. In our case, the states charged under $\mathrm{SU}(N)$ are localized near the boundary of the geometry. The fact that a global symmetry might not extend over the full bulk is not at all surprising and it happens in other situations. For example if we add $N_{f}$ massive fundamental fields, all with the same mass, to an $\mathrm{SU}(N)$ gauge theory, then we have an $\mathrm{SU}\left(N_{f}\right)$ flavor symmetry. In the gravity dual, this is realized as a brane that reaches up to a finite distance $r_{\min }$ in the bulk [42]. The larger the mass, the larger $r_{\min }$. For low energies, we explore the bulk only in the region where $r<r_{\text {min }}$ and we do not encounter states charged under the global flavor symmetry.

Here something similar is happening, the bulk $\mathrm{SU}(N)$ gauge symmetry, if present, is located only in the highly curved region, so it is not visible in the gravity region. And all bulk states that carry $\mathrm{SU}(N)$ charge, have some excitations located in that highly curved region. We can say that the $\mathrm{SU}(N)$ gauge symmetry we expected is "confined" in the bulk gravity region, but it is present in the highly curved region.

\subsection{Are there gauge fields on brane probes?}

Let us consider the ungauged model. Let us say that we have a probe D0 brane located in the region of the geometry described by Einstein gravity. Does this brane probe have a 
gauge field on its worldvolume? Naively, one would say no, since we are dealing with the ungauged model. On the other hand, we have argued that the bulk gravity region should be essentially the same for the gauged and ungauged models, so that we would expect a gauge field on the probe worldvolume.

We think that the right answer is the second, namely that there is a gauge field on the brane probes if the probes are in the Einstein gravity region, the region where $r<\lambda^{1 / 3}$. This seems to be the only reasonable answer since these are the only kinds of D0 branes that we have in the ten dimensional string theory. This gauge field imposes a constraint saying that the number of strings ending on the D0 brane should be equal to the number coming out. From the matrix model point of view, the degrees of freedom on the brane probe are effective low energy degrees of freedom that describe a complicated bound state where the matrices have large fluctuations. Recall the discussion in section 2.3. For this reason they reflect more the dynamics of these degrees of freedom than the properties of the precise UV definition of the model.

On the other hand, if we consider a D0 brane probe in the highly curved region, which is described in perturbation theory, then we do not expect to have a gauge field on the brane probe, since we do not have it in the ungauged model.

It would be interesting to understand what kind of transmutation the brane probe undergoes so that this happens as it crosses from the weakly curved bulk region to the strongly curved one.

\subsection{The ungauged model and M-theory}

In [5] the BFSS model was introduced as a tool to extract the S-matrix for 11 dimensional M-theory. In contrast to the discussion in most of this paper, the BFSS proposal is to consider a very low energy limit of this matrix model. In this very low energy limit we go very deep inside the bulk, where the 11th dimension becomes large compared to other quantities and the physics is expected to reproduce the $11 \mathrm{~d}$ one. It seems that the difference between the gauged and the ungauged model is really lost when we go to such low energies, $E \propto 1 / N$, so that we could have as well started from the ungauged model also.

\subsection{Physical realizations}

It seems that the remarks in this paper suggest that if we wanted to build a quantum computer that simulates this problem we could start with a set of harmonic oscillators and Majorana fermions and then fine tune the interactions so that we get the ungauged model. This seems simpler than producing the gauged model where the $\mathrm{SU}(N)$ gauge redundancy should emerge from some other further model. In other words, it seems simpler to try to arrange for a model having an approximate $\mathrm{SU}(N)$ global symmetry than having to produce one with the $\mathrm{SU}(N)$ gauge symmetry. Because the energy of the non-singlets is higher than that of singlets we would expect that small perturbations that break the $\mathrm{SU}(N)$ global symmetry should not be important in the IR. Still, it is important not to generate terms that lead to relevant perturbations of the model. But the number of those to fine tune seems smaller than those of all possible couplings. 


\section{Conclusions}

We have seen that the Einstein gravity region can be present in both the gauged and ungauged versions of the model. The extra degrees of freedom of the ungauged model reside in the highly curved region of the geometry. We can say that both the gauged and ungauged models flow to the same theory in the infrared. Or that the ungauged model flows to the gauged model in the IR. Of course, it is not surprising that they have something in common, since the singlet sector is common to both theories. What we wanted to highlight here was that the non-singlets do not modify the gravity solution in the region where the gravity approximation is valid.

A very similar story was found in the single matrix quantum mechanics in [8]. There the two models coincided as long as the temperature was low enough. In that case, at temperatures higher than a critical temperature the ungauged model would undergo a phase transition, somewhat reminiscent of the deconfinement transitions. See also [11] for a relation between that phase and black holes. In our case, the black hole phase is present both for the gauged and ungauged models.

We should emphasize that many of our statements can be rephrased in terms of expectations values of Wilson loops in the gauged model. We mainly talked about the non-supersymmetric Wilson loop. For example, a Wilson loop in the adjoint representation computed by a string like the one displayed in figure 1 (and extended along the time direction). This same loop has higher energy excitations where the string looks like the ones in figure 2(b), 2(c).

We have noted that the fact that the string has high tension implies that the eigenvalue distribution of the thermal holonomy, or Polyakov loop operator, has only a very small inhomogeneity when we have black hole present, see figure 4. One might have expected that the black hole formation would result in a stronger eigenvalue localization for the Polyakov loop. This is the Polyakov loop of the full model, the UV theory, which is the only one we know how to define precisely in this theory.

In the Gurau-Witten tensor models, in a sense, the opposite from what we said here happens. In such models, in the leading large $N$ approximation the basic field behaves as a conformal field with low scaling dimension. Therefore we do not have an energy gap to the non-singlets as we had in the D0 brane matrix model. In those cases the ungauged model seems a better starting point to describe the physics.

\section{Acknowledgments}

We have benefited from ongoing discussions with E. Berkowitz, M. Hanada, E. Rinaldi and P. Vranas who explained to us the details and various results of their numerical simulations of the ungauged model [7]. Those results were very helpful for us to gain confidence in the picture proposed here.

We thank I. Klebanov and J. Polchinski for discussions. J.M. is supported in part by U.S. Department of Energy grant de-sc0009988 and the Simons Foundation grant 385600. 


\section{A Details of the perturbative computations}

\section{A.1 Non singlets in the BMN matrix model}

In this appendix we will study the BMN matrix model. Lagrangian reads as follows:

$$
\begin{array}{r}
\mathcal{L}=\frac{1}{g^{2}}\left(\frac{1}{2} \sum_{I=1}^{9}\left(\dot{X}^{I}\right)^{2}-\frac{1}{2}\left(\frac{\mu}{3}\right)^{2} \sum_{a=1,2,3}\left(X^{a}\right)^{2}-\frac{1}{2}\left(\frac{\mu}{6}\right)^{2} \sum_{i \geq 4}\left(X^{i}\right)^{2}+\frac{1}{2} \psi \dot{\psi}-\frac{\mu}{8} \psi \gamma_{123} \psi+\right. \\
\left.-i \frac{1}{3} \mu g \sum_{a, b, c=1}^{3} \operatorname{Tr}\left(X^{a} X^{b} X^{c}\right) \epsilon_{a b c}+\frac{1}{4} \operatorname{Tr}\left(\left[X^{I}, X^{J}\right]^{2}\right)+i \frac{1}{2} \operatorname{Tr}\left(\psi \gamma^{I}\left[\psi, X^{I}\right]\right)\right) .
\end{array}
$$

And supersymmetry transformations are given by:

$$
\begin{aligned}
{\left[Q \epsilon, X^{I}\right] } & =\psi \gamma^{I} \epsilon(t) \\
{[Q \epsilon, \psi] } & =\left(\gamma^{I} D X^{I}+c_{I} \mu X^{I} \gamma^{I} \gamma_{123}+i \frac{1}{2}\left[X^{I}, X^{J}\right] \gamma^{I J}\right) \epsilon(t) \\
{[Q \epsilon, A] } & =\epsilon(t) \psi \\
\epsilon(t) & =e^{-\frac{1}{12} \mu \gamma_{123} t} \epsilon_{0} \\
c_{a} & =1 / 3 \quad \text { for } a=1,2,3 \quad \text { and } \quad c_{i}=-1 / 6 \text { for } i \geq 4 .
\end{aligned}
$$

Note that supersymmetry transformations are time dependent. In the supercharge we have an additional term proportional to $\mu$ :

$$
Q \epsilon=\operatorname{Tr}\left(-P^{I} \psi \gamma^{I} \epsilon-i \frac{1}{2 g^{2}}\left[X^{K}, X^{L}\right] \psi \gamma_{K L} \epsilon-\frac{\mu}{g} c_{I} X^{I} \psi \gamma^{I} \gamma_{123} \epsilon\right)
$$

Apart from the gauge transformation generator, supercharge anticommutator now also contains rotations generators $M_{\alpha \beta}$ :

$$
\begin{aligned}
\left\{Q_{\alpha}, Q_{\beta}\right\}= & 2 H \delta_{\alpha \beta}+2 \operatorname{Tr}\left(G X^{L}\right) \gamma_{\alpha \beta}^{L}+M_{\alpha \beta} \\
M_{\alpha \beta}= & -(\mu / 3) \sum_{i, j \geq 4} \operatorname{Tr}\left(X^{j} P^{i}\right)\left(\gamma_{j i} \gamma_{123}\right)_{\alpha \beta}+(2 / 3) \mu \sum_{a, b, c=1}^{3} \operatorname{Tr}\left(X^{a} P^{b}\right) \epsilon^{a b c} \gamma_{\alpha \beta}^{c} \\
& -\frac{\mu}{6 g^{2}} \sum_{i, j \geq 4} \operatorname{Tr}\left(\psi \gamma_{i j} \psi\right)\left(\gamma^{i j} \gamma^{123}\right)_{\alpha \beta}+\frac{\mu}{12 g^{2}} \sum_{a, b \in 1,2,3} \operatorname{Tr}\left(\psi \gamma_{a b} \psi\right)\left(\gamma^{a b} \gamma^{123}\right)_{\alpha \beta} .
\end{aligned}
$$

Also recall that the gauge transformation generator is given by:

$$
G=\frac{i}{2 g^{2}}\left(2\left[D_{t} X^{I}, X^{I}\right]+\left[\psi_{\alpha}, \psi_{\alpha}\right]\right) .
$$

Again, since the super charge gauge is invariant:

$$
\left[Q_{\alpha}, G\right]=0 .
$$


Hamiltonian is given by:

$$
\begin{aligned}
H=\frac{1}{g^{2}} & \operatorname{Tr}\left(g^{4} \frac{P_{I}^{2}}{2}-\frac{1}{4}\left[X^{I}, X^{J}\right]^{2}-i \frac{1}{2} \psi \gamma^{I}\left[\psi, X^{I}\right]+\frac{1}{2}\left(\frac{\mu}{3}\right)^{2} \sum_{a=1,2,3}\left(X^{a}\right)^{2}\right. \\
& \left.+\frac{1}{2}\left(\frac{\mu}{6}\right)^{2} \sum_{i \geq 4}\left(X^{i}\right)^{2}+\frac{\mu}{8} \psi \gamma_{123} \psi+i \frac{\mu}{3} \sum_{a, b, c=1}^{3} \operatorname{Tr}\left(X^{a} X^{b} X^{c}\right) \epsilon_{a b c}\right) .
\end{aligned}
$$

However since the supersymmetry transformations are time-dependent now, commutator of Hamiltonian with a supercharge is proportional to a supercharge:

$$
\left[Q_{\alpha}, H\right]=-\operatorname{Tr}\left(\psi_{\alpha} G\right)-\frac{\mu}{12} Q_{\beta} \gamma_{\beta \alpha}^{123} .
$$

As in the BFSS case, we can remove the gauge transformation generators from the SUSY algebra by imposing (3.6) and redefining Hamiltonian:

$$
H^{\text {new }}=H-\operatorname{Tr}\left(X^{1} G\right) .
$$

Now lets discuss the perturbative spectrum of this model. It would be convenient to introduce indices from the beginning of the Latin alphabet $a, b, c, \ldots$ running from 1 to 3 whereas $i, j, k, \ldots$ run from 4 to 9 . We can introduce creation-annihilation operators by:

$$
\begin{aligned}
a_{b} & =\sqrt{\frac{3}{\mu}}\left(\frac{g P_{b}}{\sqrt{2}}-i \frac{\mu}{3 \sqrt{2} g} X_{b}\right) \\
a_{i} & =\sqrt{\frac{6}{\mu}}\left(\frac{g P_{i}}{\sqrt{2}}-i \frac{\mu}{6 \sqrt{2} g} X_{i}\right)
\end{aligned}
$$

$\mathrm{SO}(6)$ sector oscillators has mass $\mu / 6$ and $\mathrm{SO}(3)$ sector has mass $\mu / 3$. Free Hamiltonian reads as:

$$
H_{0}=\frac{\mu}{3} \operatorname{Tr} a_{b}^{\dagger} a_{b}+\frac{\mu}{6} \operatorname{Tr} a_{i}^{\dagger} a_{i} .
$$

Let us concentrate on the lightest $\mathrm{SO}(6)$ sector. The leading order correction to the energy was computed in [22] to be:

$$
V_{\mathrm{eff}}^{(1)}=g^{2}\left(\frac{3}{\mu}\right)^{2}\left(N: \operatorname{Tr} a_{i}^{\dagger} a_{i}:+\frac{1}{2}: \operatorname{Tr}\left[a_{i}^{\dagger}, a_{i}\right]\left[a_{j}^{\dagger}, a_{j}\right]:-\frac{1}{2}: \operatorname{Tr}\left[a_{i}^{\dagger}, a_{j}\right]\left[a_{i}^{\dagger}, a_{j}\right]:-: \operatorname{Tr}\left[a_{i}^{\dagger}, a_{j}^{\dagger}\right]\left[a_{i}, a_{j}\right]:\right)
$$

where one has to sum over all possible indices $i, j$ ranging from 4 to 9 .

Therefore for the simplest adjoint state $a_{i}^{\dagger}|0\rangle$ the first-order correction is positive. We can rewrite the effective potential in a bit different form [22]:

$$
V_{\mathrm{eff}}^{(1)}=g^{2}\left(\frac{3}{\mu}\right)^{2}\left(\frac{1}{2}\left(: \operatorname{Tr}\left[a_{i}^{\dagger}, a^{i}\right] T^{a}:\right)\left(: \operatorname{Tr}\left[a_{i}^{\dagger}, a^{i}\right] T^{a}:\right)-\frac{1}{2}: \operatorname{Tr}\left[a_{i}^{\dagger}, a_{j}\right]\left[a_{i}^{\dagger}, a_{j}\right]:-: \operatorname{Tr}\left[a_{i}^{\dagger}, a_{j}^{\dagger}\right]\left[a_{i}, a_{j}\right]:\right) .
$$

The last two terms are exactly the 1-loop dilatation operator in $\mathcal{N}=4 \mathrm{SYM}$. One can show that the first term is zero for singlet states. For non-singlet states build from $a_{i}^{\dagger}$ its value is proportional to the number of non-contracted indices. That is, this term is proportional 
to the quadratic Casimir of the corresponding representation. To sum up, at 1-loop level the energy of non-singlets in the representation $R$ goes up:

$$
\Delta \hat{H}_{1 \text {-loop }}=\Delta \hat{H}_{\text {gauged, 1-loop }}+\frac{9 g^{2}}{2 \mu^{2}} C_{2}(R) .
$$

However, if we study the modified Hamiltonian (A.10), we have to take into account the correction coming from the operator $\operatorname{Tr}\left(X^{1} G\right)$. Second-order perturbation theory for this additional correction yields:

$$
-g^{2}\left(\frac{3}{\mu}\right)^{2}\left(: N \operatorname{Tr} a_{i}^{\dagger} a_{i}:+\frac{1}{2}: \operatorname{Tr}\left[a_{i}^{\dagger}, a_{i}\right]\left[a_{j}^{\dagger}, a_{j}\right]:\right) .
$$

This contribution completely cancels the non-singlet contribution in (A.14). It means that the theory with supersymmetric Wilson loop has a protected $\mathrm{SO}(6)$ sector, like the original theory. For example, the energy of the simplest adjoint state $a_{i}^{\dagger}|0\rangle$ is protected and is given by

$$
E=\frac{\mu}{6}
$$

\section{A.2 BFSS model}

In this appendix we will discuss the perturbaive spectrum of adjoints and derive the estimate (4.4). We consider a backround with diagonal matrices $\left\langle X^{I}\right\rangle=B^{I}=\operatorname{diag}\left(B_{1}^{I}, \ldots, B_{N}^{I}\right)$. These break the $\mathrm{SU}(N)$ symmetry, so we will have a compact manifold of Goldstone bosons. In principle we need to study the quantum mechanics on this manifold. Since $\mathrm{SU}(N)$ acts on this manifold this quantum mechanics gives rise to states charged under $\mathrm{SU}(N)$. From the analysis of the gauged model we know that there is a single uncharged state. We now want to discussed the states with $\mathrm{SU}(N)$ charges. One can obtain their spectum as follows.

We want to study the angular motion around the diaginal background $\left\langle X^{I}\right\rangle=B^{I}$. Therefore we focus on $X^{I}$ in the following form:

$$
X^{I}(t)=\mathrm{U}(t) B^{I} U^{\dagger}(t)
$$

and plug this expression into the original Lagrangian to find the effective action for $U$ :

$$
S=\frac{1}{2 g^{2}} \int d t \operatorname{Tr}\left(\left(U^{\dagger} \partial_{t} U\right)_{r}{ }^{s}\left(\vec{B}_{r}-\vec{B}_{s}\right)^{2}\left(U^{\dagger} \partial_{t} U\right)_{s}^{r}\right)
$$

where we have used a short-hand notation $\sum_{I=1}^{9}\left(B_{r}^{I}-B_{s}^{I}\right)^{2}=\left(\vec{B}_{r}-\vec{B}_{s}\right)^{2}$.

Now we need to analyse the symmetries carefully. Under the original $\mathrm{SU}(N)$ gauge transformation $L, X^{I}$ transforms as in eq. (A.18): $X^{I} \rightarrow L X^{I} L^{\dagger}$. It is equivalent to multiplying $U$ by $L$ from the left:

$$
U \rightarrow L U
$$

In other words, the original gauge group $\mathrm{SU}(N)$ acts by left rotations of $U$. Obviously, it is a symmetry of (A.19). So the states will come in $\mathrm{SU}(N)$ multiplets. The corresponding charges are given by:

$$
G_{r}{ }^{s}=\frac{1}{g^{2}} \operatorname{Tr}\left(U^{\dagger} \partial_{t} U\left[B^{I},\left[B^{I}, U^{\dagger} T_{r}{ }^{s} U\right]\right]\right)
$$


It is straightforward to check that they coinside with the charges $G$ in the Gauss law (2.2), as expected. Note that $G_{r}{ }^{s}$ is not a matrix element, but a charge corresponding to $\mathrm{SU}(N)$ algebra generator $T_{r}{ }^{s}$ which has only one non-zero element on $r$-row and $s$-column.

However, we can also multiply $U$ by a $\mathrm{SU}(N)$ matrix $R$ from the right:

$$
U \rightarrow U R \text {. }
$$

This is not a symmetry of (A.19). So the corresponding current

$$
\widetilde{G}_{r}{ }^{s}=\frac{1}{g^{2}} \operatorname{Tr}\left(U^{\dagger} \partial_{t} U\left[B^{I},\left[B^{I}, T_{r}{ }^{s}\right]\right]\right)=\frac{1}{g^{2}}\left(U^{\dagger} \partial_{t} U\right)_{r}{ }^{s}\left(\vec{B}_{r}-\vec{B}_{s}\right)^{2}
$$

does not commute with the Hamiltonian. Nonetheless, as was clarified in [8, 9] left and right multiplications of $U$ are tightly related. To understant this, let us consider a wave function $\Psi_{\mathcal{R}}^{a}\left(X^{I}\right), a=1, \ldots, \operatorname{dim} \mathcal{R}$ in some representation $\mathcal{R}$ under the gauge group. Since it lives in the representation $\mathcal{R}$ it has, by definition, the following decomposition:

$$
\Psi_{\mathcal{R}}^{a}\left(X^{I}\right)=\sum_{b=1}^{\operatorname{dim} \mathcal{R}} U_{\mathcal{R}}^{a b} \psi_{b}\left(B^{I}\right)
$$

where $U_{\mathcal{R}}^{a b}$ is the $a b$ matrix element of $U$ in the representation $\mathcal{R}$. We are interested solely in $\psi_{b}\left(B^{I}\right)$ which also lives in $\mathcal{R}$. Left $\mathrm{SU}(N)$ rotations of $U$ rotate $\Psi_{\mathcal{R}}^{a}\left(X^{I}\right)$ and $U$, leaving $\psi_{b}\left(B^{I}\right)$ invariant. Whereas right rotations transform $U$ and $\psi_{b}\left(B^{I}\right)$, leaving $\Psi_{\mathcal{R}}^{a}\left(X^{I}\right)$ invariant. Note that in both cases the representation $\mathcal{R}$ is the same. It means that charges $\widetilde{G}_{r}{ }^{s}$ act on states $\psi_{b}\left(B^{I}\right)$ by the corresponding generator $\left(T_{r}{ }^{s}\right)_{\mathcal{R}}$ in the representation $\mathcal{R}$.

What is the physical meaning of operators $\widetilde{G}_{r}^{s}$ ? One can think about $X^{I}$ as a rigid body in a space acted on by the $\mathrm{SU}(N)$ transformations. Since $\Psi_{\mathcal{R}}^{a}\left(X^{I}\right)$ stays invariant under $\widetilde{G}_{r}{ }^{s}$, they have a meaning of angular momentum operators in the frame where the body is fixed. It is well-known from the classical mechanics, that such operators are very useful for studying the rigid body motion, despite the fact that they are not conserved.

As we have just mentioned, $\tilde{G}_{r}{ }^{s}$ do not commute with the Hamiltonian. However, the Hamiltonian can be expressed in terms of them. Indeed, it is easy to see that

$$
H=\frac{g^{2}}{2} \sum_{r, s=1}^{N} \frac{\widetilde{G}_{s}^{r} \widetilde{G}_{r}{ }^{s}}{\left(\vec{B}_{s}-\vec{B}_{r}\right)^{2}} .
$$

When we focus on a particular repsentation $\mathcal{R}$, then $\widetilde{G}_{r}^{s}$ act by the $\mathrm{SU}(N)$ generator $\left(T_{r}{ }^{s}\right)_{\mathcal{R}}$ in this repsentation. The corresponding wave function depends only on $B^{I}$. This wave function is exactly what we previously called $\psi_{b}\left(B^{I}\right)$. Naively, $H$ is a $\operatorname{dim} \mathcal{R} \times \operatorname{dim} \mathcal{R}$ matrix. However, the expression for the charges in (A.23) implies that the diagonal elements $\tilde{G}_{s}{ }^{s}=0$ vanish (no sum). This implies that we have much less components. As we will see shortly, in the simplest case of the adjoint representation instead of the naive $N^{2}-1$ we will have only $N-1$ states. Generically all these states have different energies. However, we would like to emphasize that each of these $N-1$ eigenstates has a degeneracy $N^{2}-1$ (or $\operatorname{dim} \mathcal{R}$ in the generic case) because of the angular degree of freedom $U$ which we have eliminated. 
So far we have been using the canonical quantization of non-singlets. Below we will re-derive (A.25) using the path integral techniques. Moreover, the fact that we always have a degeneracy $\operatorname{dim} \mathcal{R}$ will become especially clear.

For the case of a single matrix model the result (A.25) was obtained in [8-10]. But unlike the one matrix case, we cannot diagonalize all the matrices simultaneously for generic matrix configurations. Therefore (A.25) will receive higher loop corrections from off-diagonal fluctuations of $X^{I}$ and $\psi_{\alpha}$.

There is another, more clean-cut way, how to derive eq. (A.25) which will illustrate the above points. As we have mentioned in section 3.3 if we are interested in excitations of the ungauged model in a representation $\overline{\mathcal{R}}$ (conjugate to $\mathcal{R}$ ) under $\mathrm{SU}(N)$ we can study the gauged model coupled to a Wilson line in representation $\mathcal{R}$ :

$$
\operatorname{dim} \mathcal{R} \operatorname{Tr}_{\mathcal{R}} P \exp \left(i \int d t A_{t}\right)=\operatorname{dim} \mathcal{R} \operatorname{Tr} P \exp \left(i \int d t\left(A_{t}\right)_{r}{ }^{s}\left(T_{s}{ }^{r}\right)_{\mathcal{R}}\right) .
$$

At this point it is by no means necessary to think about $A_{t}$ as a gauge field. In the ungauged case one can think about it as an auxillary Lagrange multiplier which forces the states to live in a particular representaion. Note, however, that in the ungauged model we have to multiply the Wilson loop by the dimension of the corresponding representation. This can be explained as follows. In the ungauged model we put $A_{t}$ to be zero. We can achieve this by inserting the delta function into the path integral:

$$
\delta\left(P \exp \left(i \int d t A_{t}\right)\right) .
$$

Now we can re-express the delta function in terms of characters [10]:

$$
\delta\left(P \exp \left(i \int d t A_{t}\right)\right)=\sum_{\mathcal{R}} \operatorname{dim} \mathcal{R} \operatorname{Tr}_{\mathcal{R}} P \exp \left(i \int d t A_{t}\right) .
$$

We separate $X^{I}$ into the constant background $B^{I}$ and a fluctuation $Y^{I}: X^{I}=B^{I}+Y^{I}$. Then the part of the (bosonic) action containing $A_{t}$ reads as:

$$
\frac{1}{2 g^{2}} \int d t \operatorname{Tr}\left(\partial_{t} Y^{I}+i\left[A_{t}, Y^{I}\right]+i\left[A_{t}, B^{I}\right]\right)^{2}
$$

At 1-loop level we can simply ignore $Y^{I}$ and integrate out only $A_{t}$. However at higher loops one has to take $Y^{I}$ into account. Without $Y^{I}$ we have a simple quadratic action for $A_{t}$ :

$$
-\frac{1}{2 g^{2}} \int d t\left(\vec{B}_{r}-\vec{B}_{s}\right)^{2}\left(A_{t}\right)_{r}^{s}\left(A_{t}\right)_{s}^{r}
$$

Overall, we have the following expression:

$$
\operatorname{dim} \mathcal{R} \int D A_{t} \exp \left(-\frac{1}{2 g^{2}} \int d t\left(\vec{B}_{r}-\vec{B}_{s}\right)^{2}\left(A_{t}\right)_{r}{ }^{s}\left(A_{t}\right)_{s}^{r}\right) \operatorname{Tr} P \exp \left(i \int d t\left(A_{t}\right)_{r}{ }^{s}\left(T_{s}{ }^{r}\right)_{\mathcal{R}}\right) .
$$

Integration over $A_{t}$ yields the angular potential (A.25). 
Note that because of the factor $\operatorname{dim} \mathcal{R}$, each eigenstate of the angular potential (A.25) will contribute to the partition function with degeneracy $\operatorname{dim} \mathcal{R}$.

In the adjoint case $\left(\widetilde{G}_{r}{ }^{s}\right)_{\mathcal{R}}$ acts by a commutator with $T_{r}{ }^{s}$ on the matrix $w$ in the $\mathrm{SU}(N)$ algebra. Moreover $w$ has to be diagonal, since the diagonal charges $\widetilde{G}_{s}{ }^{s}$ vanish: $w=\operatorname{diag}\left(w_{1}, \ldots, w_{N}\right)$. So we have the following eigenvalue problem ${ }^{10}$

$$
E w_{r}=\frac{g^{2}}{2} \sum_{s=1, s \neq r}^{N} \frac{w_{r}-w_{s}}{\left(\vec{B}_{r}-\vec{B}_{s}\right)^{2}}, \quad \text { with } \quad \sum_{r=1}^{N} w_{r}=0
$$

where the last constraint comes from the restriction that the diagonal matrix $w$ is in the adjoint. This Eigenvalue problem will have $N-1$ eigenstates. We could identify the potential in figure 3 as the lowest energy state of this Hamiltonian, as a function of the $\vec{B}_{s}$. In general, the eigenvalues will depend on the particular pattern of the distances $\left(\vec{B}_{r}-\vec{B}_{s}\right)^{2}$. In the next subsection we solve it for the case when a large number of vectors $\vec{B}_{s}$ is uniformly distributed on $S^{8}$.

There is another very simple case when the energy can be obtained exactly. Suppose we are considering a configuration where the $N$ vector $\vec{B}_{s}$ take only two values: $N_{1}$ are given by $\vec{B}_{1}$ and the rest, $N_{2}=N-N_{1}$, by $\vec{B}_{2}$. Equation (A.32) will be well-defined if $w_{i}$ obey the same property: there are $N_{1}$ coordinates $w_{1}$ and $N_{2}$ of $w_{2}$. Using the constraint $N_{1} w_{1}+N_{2} w_{2}=0$ we easily obtain the energy:

$$
E=\frac{\lambda}{2\left(\vec{B}_{1}-\vec{B}_{2}\right)^{2}} .
$$

The result depends only on the sum $N_{1}+N_{2}$ and not on the individual $N_{1}, N_{2}$. Also, the factor $N$ in the numerator is important: we expect that the energy of adjoint excitations will scale as $\lambda^{1 / 3}$. Indeed, the expected size of the ground state wave function is $X \approx \lambda^{1 / 3}$. This is the value of $X$ where this computation breaks down. We can then identify the energy at this value of $X$ as the order of magnitude of the energy of the adjoint excitation

$$
E_{\text {adj }} \approx \lambda / X^{2}=\lambda^{1 / 3} .
$$

\section{A.2.1 Solving the potential for a uniform distribution}

There is another case when we can solve (A.32) exactly. Namely, lets consider the large $N$ limit with the $N$ vectors $\vec{B}_{s}$ uniformly distributed on $S^{8}$ of radius $X$. We will show below that the lowest energy state has energy

$$
E_{1}=\frac{9 \lambda}{28 X^{2}}
$$

and we will further compute spectrum around the ground state.

This is shown as follows. With a large number of vectors uniformly distributed on $S^{8}$ we can make a continous approximation. Then eq. (A.32) becomes

$$
E w(\vec{n})=\frac{\lambda}{2 X^{2} \operatorname{Vol}_{\mathrm{S}^{8}}} \int d \Omega_{8}^{\prime} \frac{w(\vec{n})-w\left(\vec{n}^{\prime}\right)}{\left|\vec{n}-\vec{n}^{\prime}\right|^{2}}, \quad \int d \Omega_{8} w(\vec{n})=0
$$

\footnotetext{
${ }^{10}$ This can be easily obtained using the following relations $\left[T_{r}{ }^{s}, w\right]=\left(w_{s}-w_{r}\right) T_{r}{ }^{s}$, and also $T_{r}{ }^{s} T_{r}{ }^{s}=$ $0, r \neq s$ and $\left(T_{r}{ }^{s} T_{s}{ }^{r}\right)_{v o}=\delta_{r v} \delta_{r o}$ (no sum over $r, s$ ).
} 
where $\vec{n}$ and $\vec{n}^{\prime}$ belong to a $S^{8}$ of unit radius. Now the adjoint problem (A.36) has $\mathrm{SO}(9)$ rotation symmetry. It means that the eigenfunctions are basically given by the spherical harmonics in nine dimensions and the energy depends only on the total angular momentum $l$. It is the most convenient to evaluate the energy using the wave function which depends only on one polar angle $\theta$ (the angle between the unit vector and $X^{9}$ axis). For such functions the measure $d y$ reads as $\operatorname{Vol}_{S^{7}} \sin ^{7} \theta d \theta=\operatorname{Vol}_{S^{7}}\left(1-t^{2}\right)^{3} d t$. In this case $w(\vec{n})$ is simply the Gegenbauer polynomial $C_{l}^{(7 / 2)}(t)$. Therefore,

$$
E_{l} C_{l}^{(7 / 2)}(1)=\frac{35 \lambda}{64 X^{2}} \int_{-1}^{1} d t \frac{\left(1-t^{2}\right)^{3}}{2(1-t)}\left(C_{l}^{(7 / 2)}(1)-C_{l}^{(7 / 2)}(t)\right)
$$

which leads to the following energies:

$$
E_{l}=\frac{3 \lambda}{8 X^{2}}\left(1-\frac{1}{C_{l}^{(7 / 2)}(1)}\right) \sim \frac{3 \lambda}{4 X^{2}}\left(1-\frac{1}{7 l^{6}}\right), \quad l \gg 1 .
$$

This energy comes with a degeneracy

$$
N_{l}=\frac{(2 l+7)(l+6) !}{7 ! l !} \sim \frac{2 l^{7}}{7 !}, \quad l \gg 7 .
$$

It is interesting that we get a finite range of energies, from a minimum one to a maximum. This pattern is similar to what we get by a simple WKB quantization of a toy model for the motion of a folded string in appendix B. However, in the gravity case, we can also have the possibility of the string falling into the black hole which leads to a much larger number of states, a number proportional to $N^{2}$, one factor of $N$ each for the separate string and anti-string ending on the black hole.

\section{A.3 Goldstone modes and $\mathrm{SU}(N)$ rotators for the BMN model vacua}

Now let us discuss the spectrum around other vacua, where the matrices have non-zero expectation values of the form $X^{a}=\frac{\mu}{3} J^{a}$. This case can be analyized as in the previous section. The only difference is that the initial action is

$$
S=\frac{\mu^{2}}{18 g^{2}} \int d t \sum_{a=1}^{3} \operatorname{Tr}\left[J^{a}, U^{\dagger} \partial_{t} U\right]^{2}=\frac{\mu^{2}}{18 g^{2}} \int d t \sum_{a=1}^{3} \operatorname{Tr} U^{\dagger} \partial_{t} U\left[J^{a},\left[J^{a}, U^{\dagger} \partial_{t} U\right]\right] .
$$

And the right $\mathrm{SU}(N)$ charge equals:

$$
\widetilde{G}^{p}=\frac{\mu^{2}}{9 g^{2}} \int d t \sum_{a=1}^{3} \operatorname{Tr}\left(\left[J^{a},\left[J^{a}, U^{\dagger} \partial_{t} U\right]\right] T^{p}\right)
$$

with $T^{p}, p=1, \ldots, N^{2}-1$ belonging to $\mathrm{SU}(N)$ algebra.

As we have mentioned in the main text, $J^{a}$ are not neccessary in the irreducible representation. Generically, we need to decompose it into $L$ irreducible representations of dimensions $N_{k}, k=1, \ldots, L$ such that $N_{1}+\ldots+N_{L}=N$. For simplicity we study the maximal representation $L=1$ and $N_{1}=N$, although the calculation below can be 
generalized to $L>1$ case. Even for the maximal case, when we have only one representation it is quite difficult to obtain the exact spectum. However, it is easy to find a sensible lower bound.

Since we have only the kinetic term the energy equals:

$$
E=\frac{\mu^{2}}{18 g^{2}} \operatorname{Tr} \int d t \sum_{a=1}^{3}\left[J^{a}, U^{\dagger} \partial_{t} U\right]^{2} .
$$

Generically, there are many ways to select Lie algebra generators $T^{p}$. However, there is a very special choice of $T^{p}$, namely the fuzzy spherical harmonics $Y_{m}^{j}, j=1, \ldots, N-1, m=$ $-j, \ldots, j$. The nice thing about them is that they are eigenvalues of the fuzzy sphere Laplacian:

$$
\sum_{a=1}^{3}\left[J^{a},\left[J^{a}, Y_{m}^{j}\right]\right]=j(j+1) Y_{m}^{j}
$$

Also they are orhogonal:

$$
\operatorname{Tr}\left(Y_{m}^{j} Y_{m^{\prime}}^{j^{\prime}}\right)=\frac{1}{2} \delta_{j j^{\prime}} \delta_{-m m^{\prime}} .
$$

Because of that they also satisfy the completness relation:

$$
\sum_{j m}\left(Y_{m}^{j}\right)_{r}^{s}\left(Y_{-m}^{j}\right)_{v}^{o}=\frac{1}{2}\left(\delta_{r}^{o} \delta_{v}^{s}-\frac{1}{N} \delta_{r}^{o} \delta_{v}^{s}\right), \quad r, s, v, o=1, \ldots, N
$$

Correspondingly we have the non-conserved charges $\widetilde{G}_{m}^{j}$ :

$$
\widetilde{G}_{m}^{j}=j(j+1) \frac{\mu^{2}}{9 g^{2}} \operatorname{Tr}\left(U^{\dagger} \partial_{t} U Y_{m}^{j}\right)
$$

Finally, we can rewrite the Hamiltonian in terms of $\widetilde{G}_{m}^{j}$ using eq. (A.45):

$$
\begin{aligned}
H & =\frac{\mu^{2}}{9 g^{2}} \sum_{a=1}^{3} \sum_{j m} \operatorname{Tr}\left(U^{\dagger} \partial_{t} U Y_{m}^{j}\right) \operatorname{Tr}\left(Y_{-m}^{j}\left[J^{a},\left[J^{a}, U^{\dagger} \partial_{t} U\right]\right]\right) \\
& =\frac{\mu^{2}}{9 g^{2}} \sum_{j m} j(j+1) \operatorname{Tr}\left(U^{\dagger} \partial_{t} U Y_{m}^{j}\right) \operatorname{Tr}\left(Y_{-m}^{j} U^{\dagger} \partial_{t} U\right) \\
& =\frac{9 g^{2}}{\mu^{2}} \sum_{j m} \frac{\widetilde{G}_{m}^{j} \widetilde{G}_{-m}^{j}}{j(j+1)} .
\end{aligned}
$$

As we have promised, we have re-expressed the Hamiltonian in terms of charges $\widetilde{G}$. If we focus on some particular representation $\mathcal{R}$, then $\widetilde{G}_{m}^{j}$ act as Lie algebra generators $\left(Y_{m}^{j}\right)_{\mathcal{R}}$ in this representaion. For example, the sum $\sum_{j m} \widetilde{G}_{m}^{j} \widetilde{G}_{-m}^{j}=C_{2}(\mathcal{R})$ equals to the quadratic Casimir of the representation. Since $j \leq N-1$ we obtain the following lower bound for the energy:

$$
H \geq \frac{9 g^{2}}{\mu^{2}} \frac{1}{N(N-1)} C_{2}(\mathcal{R})
$$


The above derivation can be repeated when we have several fuzzy spheres with corresponding representations $N_{k}$. In this case one arrives at the following bound:

$$
H \geq \frac{1}{\max N_{k}\left(N_{k}-1\right)} \frac{9 g^{2}}{\mu^{2}} C_{2}(\mathcal{R}) .
$$

For the adjoint representation of $\mathrm{SU}(N)$ the quadratic Casimir $C_{2}(\operatorname{adj})$ is simply $N$. The other representations that appear are those that can arise from products of adjoints. These are the representations that transform trivially under the $Z_{N}$ center of $\mathrm{SU}(N)$.

For "small" fuzzy spheres, when $N_{k} \sim \mathcal{O}\left(N^{0}\right)$ and $L \sim \mathcal{O}(N), E_{\text {adj }} \gtrsim \lambda / \mu^{2}$. However, if we have a "big" sphere, when some $N_{k} \sim N$ and so $L \sim 1$, adjoints can have much smaller energy $E_{\text {adj }} \geq \frac{g^{2}}{N \mu^{2}}$. Note that both these bounds are consistent with

$$
E \sim \frac{g^{2} N}{R^{2}} C_{2}(\mathcal{R})
$$

with $R^{2}=\frac{1}{3 N} \operatorname{Tr}\left(J_{1}^{2}+J_{2}^{2}+J_{3}^{2}\right)$. Since for each irreducible representation $N_{k}$ we have the following identity:

$$
J_{1}^{2}+J_{2}^{2}+J_{3}^{2}=\frac{N_{k}^{2}-1}{4} \mathbf{1}
$$

where the right hand side is simply the quadratic Casimir of $\mathrm{SU}(2)$ in the representation of dimension $N_{k}$.

(A.50) is what we would have naively guessed based on the similar formula for the case of diagonal matrices $X$ that we derived in appendix A.2, and was mentioned in (4.4).

\section{B Analyzing the motion of a folded string}

In this appendix we consider the motion of a folded stretched string. This is just a one parameter family of solutions out of the whole space of possible string motions.

We can view the motion of the tip from the high curvature region to the low curvature and back as a kind of scattering problem. See figure 5. So we can calculate the total phase shift accumulated during the process via a WKB approximation.

We view the system as the tip of a string which is approximated as a particle with large momentum $p$ and energy linear in the momentum. This tip is acted on by the rest of the string which provides a potential. The full Hamiltonian is

$$
H=\sqrt{-\frac{g_{t t}}{g_{r r}}}|p|+\frac{r_{\infty}-r}{\pi}
$$

where $p$ is the momentum conjugate to $r$. The tip of the string starts from the large $r$ region with very high ingoing radial momentum, the string pulls and slows it down until the tip bounces back to the large $r$ region, see figure 5 . The total phase shift then is

$$
\delta_{\mathrm{bulk}}=2 \int_{r_{\infty}-\pi E}^{r_{\infty}} d r p
$$


Which can be re-written in terms of the total energy $E$ given in (B.1). This gives for $E \rightarrow \frac{r_{\infty}}{\pi}:$

$$
\delta_{\text {bulk }}=\frac{8 \sqrt{\lambda d_{0}}}{15 \pi\left(\pi E_{\infty}-\pi E_{n}\right)^{3 / 2}} .
$$

Where $E_{\infty} \equiv \tilde{C} \lambda^{1 / 3}$ is the energy of a folded string that stretches all the way to $r=0$. For this reason we expect that $\tilde{C}>C$ by an order one amount. A similar problem in the linear dilaton background that is dual to the double scaling limit of a single matrix model was analyzed in [12] and matched to the matrix model computation in [13].

In order to figure out the whole motion, we need to know how the tip bounces back from the high curvature region. This seems to be a difficult problem since the state that comes out could have more excitations on the string worldvolume. Solving this would involve connecting the motion in the weakly curved region to the motion in the perturbative matrix model region. We will not do this here. Instead we will simply assume that the problem is such that the string tip comes back out with an extra phase shift $\delta_{\text {high }}$ from the high curvature region. Furthermore we will assume that it is basically a constant for $E \sim E_{\infty}$. This is a non-trivial assumption and it is likely wrong. The only reason we make it is to define a toy problem where we can now semiclassically quantize the motion by setting

$$
\delta_{\text {bulk }}(E)+\delta_{\text {high }}=2 \pi n .
$$

Leading to

$$
E_{n} \sim \lambda^{1 / 3}\left[\widetilde{C}-\frac{1}{\pi}\left(\frac{4 \sqrt{d_{0}}}{15 \pi^{2}\left(n-n_{0}\right)}\right)^{2 / 3}\right], \quad n_{0} \equiv \frac{\delta_{\text {high }}}{2 \pi} .
$$

Note that $n_{0}$ is not an integer.

We see that there is an infinite tower of excitations. For non-zero temperature, there is actually an $n_{\max }-n_{0} \propto T^{-3 / 5}$ where the states change behavior qualitatively, the folded string falls into the black hole horizon and stays there forever. (At finite $N$ the string can break and the fold can return to infinity). To describe this behavior we need to study the non-extermal metric (2.7). Now we have a non-extremal black hole with a horizon at $r=r_{0}$. If the string has enough energy to reach the horizon, then the tip will fall into the black hole and never come back. This sets an upper bound for the energy:

$$
E_{\mathrm{dec}}-E_{\infty}=-\frac{r_{0}}{\pi}
$$

Moreover now we have a finite number of excited states that do not fall into the black hole

$$
n_{\max }-n_{0}=\sqrt{\lambda d_{0}} \int_{r_{0}}^{r_{\infty}} d r \frac{r-r_{0}}{\pi^{2} r^{7 / 2}\left(1-r_{0}^{7} / r^{7}\right)}=\frac{4.06 \sqrt{\lambda d_{0}}}{14 \pi^{2} r_{0}^{3 / 2}} \sim T^{-3 / 5}, \quad \text { for } \quad r_{\infty} \gg r_{0} .
$$

For states with $E>E_{\mathrm{dec}}$, the string tip falls into the black hole and the state becomes a string and an anti-string, both ending at the horizon as independent excitations. 


\section{Scaling properties of the solution and the action}

In this appendix we briefly discuss some scaling properties of the solution (2.7). We find that under the following rescaling of the coordinates the metric and the dilaton rescale as

$$
\begin{aligned}
t & \rightarrow \eta t, & r & \rightarrow \eta^{-2 / 5} r \\
d s^{2} & \rightarrow d s^{2} \eta^{-3 / 5}, & e^{2 \phi} & \rightarrow e^{2 \phi} \eta^{-21 / 5} .
\end{aligned}
$$

The gravity action scales as

$$
S_{\text {gravity }}=\int d^{10} x e^{-2 \phi} \sqrt{g} R \sim \eta^{-9 / 5} \sim T^{9 / 5} .
$$

Notice that $\beta$ is recaled when we rescale time. This is the correct behaviour of the Bekenstein-Hawking entropy (2.11). Notice that the action and entropy scale in the same way. Notice that since the action changes (C.1) is not a symmetry of the action, but it helps determine the temperature dependence.

It turns out that the Dirac-Born-Infeld(DBI) action for a probe D0 brane in the extremal geometry (2.7) with $r_{0}=0$ has exactly the same scaling behaviour. This can be checked explicitly, but we can also derive it by the following observations. The action is

$$
S_{\mathrm{DBI}}=-\int e^{-\phi} d s+\int A_{t} d t
$$

We now observe:

- The derivative of the free energy with respect to the charge yields the difference between the RR 1-form at the horizon and infinity: ${ }^{11}$

$$
\frac{\partial F}{\partial N}=\left.A_{t}\right|_{\text {horizon }}-\left.A_{t}\right|_{\text {infinity }}
$$

this is why $A_{t}$ scales as the free energy.

- Notice that the expression for $A_{t}$ in (2.7) does not contain $r_{0}$. This is why it has exactly the same scaling for both extremal and non-extremal cases.

- Finally, both terms in (C.3) scale in the same way as in the extremal case because of the supersymmetry (there should be no force acting on a D0 brane at rest).

Now, this observation also explains why the following action has the same rescaling properties

$$
S=\int d t\left[\vec{v}_{i}^{2}+(\text { const. }) \frac{\left(\vec{v}_{i}-\vec{v}_{j}\right)^{4}}{\left|\vec{r}_{i}-r_{j}\right|^{7}}\right]
$$

under (C.1). The reason is that the velocity expansion of (C.3) gives rise to a particular case of this action.

The point of these observations is to "explain" the observation in $[43,44]$ that (C.5) has the same scaling as the entropy. The arguments used in that paper were scaling arguments, and they have reproduced the entropy for simple scaling reasons. But it seems that the thermodynamics of (C.5) is really ill defined because it has a "fall to the center" instability.

\footnotetext{
${ }^{11}$ More precisely, one has to substract the zero-temperature value in order to make this expression finite.
} 
Open Access. This article is distributed under the terms of the Creative Commons Attribution License (CC-BY 4.0), which permits any use, distribution and reproduction in any medium, provided the original author(s) and source are credited.

\section{References}

[1] J.M. Maldacena, The Large $N$ limit of superconformal field theories and supergravity, Int. J. Theor. Phys. 38 (1999) 1113 [hep-th/9711200] [inSPIRE].

[2] S.S. Gubser, I.R. Klebanov and A.M. Polyakov, Gauge theory correlators from noncritical string theory, Phys. Lett. B 428 (1998) 105 [hep-th/9802109] [INSPIRE].

[3] E. Witten, Anti-de Sitter space and holography, Adv. Theor. Math. Phys. 2 (1998) 253 [hep-th/9802150] [INSPIRE].

[4] B. de Wit, J. Hoppe and H. Nicolai, On the Quantum Mechanics of Supermembranes, Nucl. Phys. B 305 (1988) 545 [INSPIRE].

[5] T. Banks, W. Fischler, S.H. Shenker and L. Susskind, $M$ theory as a matrix model: A Conjecture, Phys. Rev. D 55 (1997) 5112 [hep-th/9610043] [InSPIRE].

[6] N. Itzhaki, J.M. Maldacena, J. Sonnenschein and S. Yankielowicz, Supergravity and the large $N$ limit of theories with sixteen supercharges, Phys. Rev. D 58 (1998) 046004 [hep-th/9802042] [INSPIRE].

[7] E. Berkowitz, M. Hanada, E. Rinaldi and P. Vranas, Gauged And Ungauged: A Nonperturbative Test, arXiv:1802.02985 [INSPIRE].

[8] D.J. Gross and I.R. Klebanov, Vortices and the nonsinglet sector of the $c=1$ matrix model, Nucl. Phys. B 354 (1991) 459 [inSPIRE].

[9] G. Marchesini and E. Onofri, Planar Limit for SU(N) Symmetric Quantum Dynamical Systems, J. Math. Phys. 21 (1980) 1103 [InSPIRE].

[10] D. Boulatov and V. Kazakov, One-dimensional string theory with vortices as the upside down matrix oscillator, Int. J. Mod. Phys. A 8 (1993) 809 [hep-th/0012228] [inSPIRE].

[11] V. Kazakov, I.K. Kostov and D. Kutasov, A Matrix model for the two-dimensional black hole, Nucl. Phys. B 622 (2002) 141 [hep-th/0101011] [INSPIRE].

[12] J.M. Maldacena, Long strings in two dimensional string theory and non-singlets in the matrix model, JHEP 09 (2005) 078 [hep-th/0503112] [INSPIRE].

[13] L. Fidkowski, Solving the eigenvalue problem arising from the adjoint sector of the $c=1$ matrix model, hep-th/0506132 [INSPIRE].

[14] R. Gurau and J.P. Ryan, Colored Tensor Models - a review, SIGMA 8 (2012) 020 [arXiv: 1109.4812] [INSPIRE].

[15] E. Witten, An SYK-Like Model Without Disorder, arXiv:1610.09758 [INSPIRE].

[16] E. Witten, Anti-de Sitter space, thermal phase transition and confinement in gauge theories, Adv. Theor. Math. Phys. 2 (1998) 505 [hep-th/9803131] [INSPIRE].

[17] O. Aharony, J. Marsano, S. Minwalla, K. Papadodimas and M. Van Raamsdonk, The Hagedorn/deconfinement phase transition in weakly coupled large $N$ gauge theories, in proceedings of the 5th International Workshop on Lie Theory and Its Applications in Physics (LT-5), Varna, Bulgaria, 16-22 June 2003, p. 161 [Adv. Theor. Math. Phys. 8 (2004) 603] [hep-th/0310285] [INSPIRE]. 
[18] J. Polchinski, $M$ theory and the light cone, Prog. Theor. Phys. Suppl. 134 (1999) 158 [hep-th/9903165] [INSPIRE].

[19] D.E. Berenstein, J.M. Maldacena and H.S. Nastase, Strings in flat space and pp waves from $N=4$ superYang-Mills, JHEP 04 (2002) 013 [hep-th/0202021] [INSPIRE].

[20] K. Dasgupta, M.M. Sheikh-Jabbari and M. Van Raamsdonk, Matrix perturbation theory for M-theory on a PP wave, JHEP 05 (2002) 056 [hep-th/0205185] [INSPIRE].

[21] J.M. Maldacena, M.M. Sheikh-Jabbari and M. Van Raamsdonk, Transverse five-branes in matrix theory, JHEP 01 (2003) 038 [hep-th/0211139] [INSPIRE].

[22] N. Kim, T. Klose and J. Plefka, Plane wave matrix theory from $N=4$ super-Yang-Mills on $R \times S^{3}$, Nucl. Phys. B 671 (2003) 359 [hep-th/0306054] [INSPIRE].

[23] G.T. Horowitz and A. Strominger, Black strings and P-branes, Nucl. Phys. B 360 (1991) 197 [INSPIRE].

[24] A.W. Peet and J. Polchinski, UV/IR relations in AdS dynamics, Phys. Rev. D 59 (1999) 065011 [hep-th/9809022] [INSPIRE].

[25] K.N. Anagnostopoulos, M. Hanada, J. Nishimura and S. Takeuchi, Monte Carlo studies of supersymmetric matrix quantum mechanics with sixteen supercharges at finite temperature, Phys. Rev. Lett. 100 (2008) 021601 [arXiv:0707.4454] [INSPIRE].

[26] D.N. Kabat, G. Lifschytz and D.A. Lowe, Black hole thermodynamics from calculations in strongly coupled gauge theory, in proceedings of the Strings 2000, Ann Arbor, Michigan, U.S.A., 10-15 July 2000, p. 216 [Phys. Rev. Lett. 86 (2001) 1426] [Int. J. Mod. Phys. A 16 (2001) 856] [hep-th/0007051] [INSPIRE].

[27] M. Hanada, Y. Hyakutake, J. Nishimura and S. Takeuchi, Higher derivative corrections to black hole thermodynamics from supersymmetric matrix quantum mechanics, Phys. Rev. Lett. 102 (2009) 191602 [arXiv: 0811.3102] [INSPIRE].

[28] D. Kadoh and S. Kamata, Gauge/gravity duality and lattice simulations of one dimensional SYM with sixteen supercharges, arXiv:1503.08499 [INSPIRE].

[29] S. Catterall and T. Wiseman, Black hole thermodynamics from simulations of lattice Yang-Mills theory, Phys. Rev. D 78 (2008) 041502 [arXiv:0803.4273] [INSPIRE].

[30] V.G. Filev and D. O'Connor, The BFSS model on the lattice, JHEP 05 (2016) 167 [arXiv: 1506.01366] [INSPIRE].

[31] E. Berkowitz, E. Rinaldi, M. Hanada, G. Ishiki, S. Shimasaki and P. Vranas, Precision lattice test of the gauge/gravity duality at large- $N$, Phys. Rev. D 94 (2016) 094501 [arXiv: 1606.04951] [INSPIRE].

[32] H. Lin and J.M. Maldacena, Fivebranes from gauge theory, Phys. Rev. D 74 (2006) 084014 [hep-th/0509235] [INSPIRE].

[33] M.S. Costa, L. Greenspan, J. Penedones and J. Santos, Thermodynamics of the BMN matrix model at strong coupling, JHEP 03 (2015) 069 [arXiv:1411.5541] [INSPIRE].

[34] Y. Asano, G. Ishiki, T. Okada and S. Shimasaki, Emergent bubbling geometries in the plane wave matrix model, JHEP 05 (2014) 075 [arXiv:1401.5079] [INSPIRE].

[35] Y. Asano, G. Ishiki, S. Shimasaki and S. Terashima, Spherical transverse M5-branes in matrix theory, Phys. Rev. D 96 (2017) 126003 [arXiv:1701.07140] [INSPIRE]. 
[36] S.-J. Rey and J.-T. Yee, Macroscopic strings as heavy quarks in large $N$ gauge theory and anti-de Sitter supergravity, Eur. Phys. J. C 22 (2001) 379 [hep-th/9803001] [INSPIRE].

[37] J.M. Maldacena, Wilson loops in large N field theories, Phys. Rev. Lett. 80 (1998) 4859 [hep-th/9803002] [INSPIRE].

[38] L.F. Alday and J.M. Maldacena, Comments on gluon scattering amplitudes via AdS/CFT, JHEP 11 (2007) 068 [arXiv:0710.1060] [INSPIRE].

[39] J. Polchinski and J. Sully, Wilson Loop Renormalization Group Flows, JHEP 10 (2011) 059 [arXiv: 1104.5077] [INSPIRE].

[40] G.T. Horowitz and E.J. Martinec, Comments on black holes in matrix theory, Phys. Rev. D 57 (1998) 4935 [hep-th/9710217] [INSPIRE].

[41] R. Gregory and R. Laflamme, Black strings and p-branes are unstable, Phys. Rev. Lett. 70 (1993) 2837 [hep-th/9301052] [INSPIRE].

[42] A. Karch and E. Katz, Adding flavor to AdS/CFT, JHEP 06 (2002) 043 [hep-th/0205236] [INSPIRE].

[43] A.V. Smilga, Comments on thermodynamics of supersymmetric matrix models, Nucl. Phys. B 818 (2009) 101 [arXiv: 0812.4753] [INSPIRE].

[44] T. Morita, S. Shiba, T. Wiseman and B. Withers, Warm p-soup and near extremal black holes, Class. Quant. Grav. 31 (2014) 085001 [arXiv:1311.6540] [INSPIRE]. 